\title{
Zero-one Schubert polynomials
}

\author{
Alex Fink ${ }^{1} \cdot$ Karola Mészáros ${ }^{2,3} \cdot$ Avery St. Dizier $^{2}$ \\ Received: 2 September 2019 / Accepted: 5 April 2020 / Published online: 10 June 2020 \\ (c) The Author(s) 2020
}

\begin{abstract}
We prove that if $\sigma \in S_{m}$ is a pattern of $w \in S_{n}$, then we can express the Schubert polynomial $\mathfrak{S}_{w}$ as a monomial times $\mathfrak{S}_{\sigma}$ (in reindexed variables) plus a polynomial with nonnegative coefficients. This implies that the set of permutations whose Schubert polynomials have all their coefficients equal to either 0 or 1 is closed under pattern containment. Using Magyar's orthodontia, we characterize this class by a list of twelve avoided patterns. We also give other equivalent conditions on $\mathfrak{S}_{w}$ being zero-one. In this case, the Schubert polynomial $\mathfrak{S}_{w}$ is equal to the integer point transform of a generalized permutahedron.
\end{abstract}

\section{Introduction}

Schubert polynomials, introduced by Lascoux and Schützenberger in [10], represent cohomology classes of Schubert cycles in the flag variety. Knutson and Miller also showed them to be multidegrees of matrix Schubert varieties [7]. There are a number of combinatorial formulas for the Schubert polynomials $[1,2,5,6,9,12,14,17]$, yet only recently has the structure of their supports been investigated: the support of a Schubert polynomial $\mathfrak{S}_{w}$ is the set of all integer points of a certain generalized permutahedron $\mathcal{P}(w)$ [4,15]. The question motivating

Fink was partially supported by an Engineering and Physical Sciences Research Council grant (EP/M01245X/1), and is now supported by the European Union's Horizon 2020 research and innovation programme under grant agreement No 792432. Mészáros is partially supported by a National Science Foundation Grant (DMS 1501059), as well as by a von Neumann Fellowship at the IAS funded by the Fund for Mathematics and Friends of the Institute for Advanced Study.

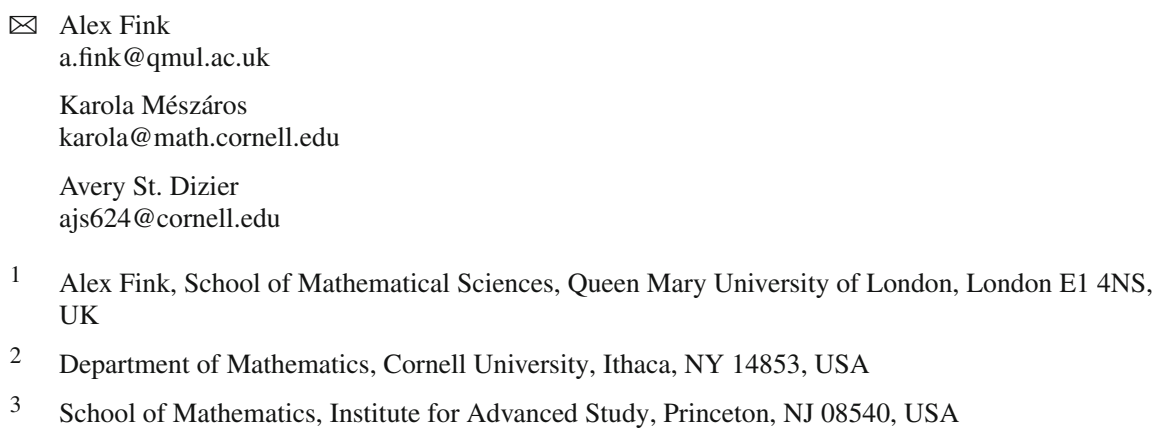


this paper is to characterize when $\mathfrak{S}_{w}$ equals the integer point transform of $\mathcal{P}(w)$, in other words, when all the coefficients of $\mathfrak{S}_{w}$ are equal to 0 or 1 .

One of our main results is a pattern-avoidance characterization of the permutations corresponding to these polynomials:

Theorem 1.1 The Schubert polynomial $\mathfrak{S}_{w}$ is zero-one if and only if $w$ avoids the patterns 12543, 13254, 13524, 13542, 21543, 125364, 125634, 215364, 215634, 315264, 315624, and 315642 .

In Theorem 4.8 we provide further equivalent conditions on the Schubert polynomial $\mathfrak{S}_{w}$ being zero-one. One implication of Theorem 1.1 follows from our other main result, which relates the Schubert polynomials $\mathfrak{S}_{\sigma}$ and $\mathfrak{S}_{w}$ when $\sigma$ is a pattern of $w$ :

Theorem 1.2 Fix $w \in S_{n}$ and let $\sigma \in S_{n-1}$ be the pattern with Rothe diagram $D(\sigma)$ obtained by removing row $k$ and column $w_{k}$ from $D(w)$. Then

$$
\mathfrak{S}_{w}\left(x_{1}, \ldots, x_{n}\right)=M\left(x_{1}, \ldots, x_{n}\right) \mathfrak{S}_{\sigma}\left(x_{1}, \ldots, \widehat{x_{k}}, \ldots, x_{n}\right)+F\left(x_{1}, \ldots, x_{n}\right),
$$

where $F \in \mathbb{Z}_{\geq 0}\left[x_{1}, \ldots, x_{n}\right]$ and

$$
M\left(x_{1}, \ldots, x_{n}\right)=\left(\prod_{(k, i) \in D(w)} x_{k}\right)\left(\prod_{\left(i, w_{k}\right) \in D(w)} x_{i}\right) .
$$

Theorem 1.2 is a special case of Theorem 5.8, which applies to the dual character of the flagged Weyl module of any diagram.

\section{Outline of this paper}

Section 2 gives an expression of Magyar for Schubert polynomials in terms of orthodontic sequences $(\boldsymbol{i}, \boldsymbol{m})$. In Sect. 3, we give a condition "multiplicity-free" on the orthodontic sequence $(\boldsymbol{i}, \boldsymbol{m})$ of $w$ which implies that $\mathfrak{S}_{w}$ is zero-one. In Sect. 4 we show that multiplicityfreeness can equivalently be phrased in terms of pattern avoidance. We then prove in Sect. 4 that multiplicity-freeness is also a necessary condition for $\mathfrak{S}_{w}$ to be zero-one. In the latter proof we assume Theorem 1.2, whose generalization (Theorem 5.8) and proof is the subject of Sect. 5.

\section{Magyar's orthodontia for Schubert polynomials}

In this section we explain the results we use to show one direction of Theorem 1.1. We include the classical definition of Schubert polynomials here for reference.

The Schubert polynomial of the longest permutation $w_{0}=n n-1 \cdots 21 \in S_{n}$ is

$$
\mathfrak{S}_{w_{0}}:=x_{1}^{n-1} x_{2}^{n-2} \cdots x_{n-1} .
$$

For $w \in S_{n}, w \neq w_{0}$, there exists $i \in[n-1]$ such that $w_{i}<w_{i+1}$. For any such $i$, the Schubert polynomial $\mathfrak{S}_{w}$ is defined as

$$
\mathfrak{S}_{w}\left(x_{1}, \ldots, x_{n}\right):=\partial_{i} \mathfrak{S}_{w s_{i}}\left(x_{1}, \ldots, x_{n}\right),
$$

where $s_{i}$ is the transposition swapping $i$ and $i+1$, and $\partial_{i}$ is the $i$ th divided difference operator

$$
\partial_{i}(f):=\frac{f\left(x_{1}, \ldots, x_{n}\right)-f\left(x_{1}, \ldots, x_{i-1}, x_{i+1}, x_{i}, x_{i+2}, \ldots, x_{n}\right)}{x_{i}-x_{i+1}} .
$$


Since the operators $\partial_{i}$ satisfy the braid relations, the Schubert polynomials $\mathfrak{S}_{w}$ are welldefined.

We will not be using the above definition of Schubert polynomials in this work. Instead, we will make use of several results due to Magyar in [13]. We start by summarizing Proposition 15 and Proposition 16 of [13] and supplying the necessary background, closely following the exposition of [13].

By a diagram, we mean a sequence $D=\left(C_{1}, C_{2}, \ldots, C_{n}\right)$ of finite subsets of $[n]$, called the columns of $D$. We interchangeably think of $D$ as a collection of boxes $(i, j)$ in a grid, viewing an element $i \in C_{j}$ as a box in row $i$ and column $j$ of the grid. When we draw diagrams, we read the indices as in a matrix: $i$ increases top-to-bottom and $j$ increases leftto-right. Two diagrams $D$ and $D^{\prime}$ are called column-equivalent if one is obtained from the other by reordering nonempty columns and adding or removing any number of empty columns. For a column $C \subseteq[n]$, let the multiplicity mult ${ }_{D}(C)$ be the number of columns of $D$ which are equal to $C$. The sum of diagrams, denoted $D \oplus D^{\prime}$, is constructed by concatenating the lists of columns; graphically this means placing $D^{\prime}$ to the right of $D$.

The Rothe diagram $D(w)$ of a permutation $w \in S_{n}$ is the diagram

$$
D(w)=\left\{(i, j) \in[n] \times[n] \mid i<\left(w^{-1}\right)_{j} \text { and } j<w_{i}\right\} .
$$

Note that Rothe diagrams have the northwest property: If $\left(r, c^{\prime}\right),\left(r^{\prime}, c\right) \in D(w)$ with $r<r^{\prime}$ and $c<c^{\prime}$, then $(r, c) \in D(w)$.

Example 2.1 If $w=31542$, then

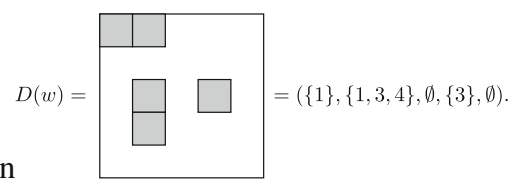

We next recall Magyar's orthodontia. Let $D$ be the Rothe diagram of a permutation $w \in S_{n}$ with columns $C_{1}, C_{2}, \ldots, C_{n}$. We describe an algorithm for constructing a reduced word $\boldsymbol{i}=\left(i_{1}, \ldots, i_{l}\right)$ and a multiplicity list $\boldsymbol{m}=\left(k_{1}, \ldots, k_{n} ; m_{1}, \ldots, m_{l}\right)$ such that the diagram $D_{i, m}$ defined by

$$
D_{\boldsymbol{i}, \boldsymbol{m}}=\bigoplus_{j=1}^{n} k_{j} \cdot[j] \oplus \bigoplus_{j=1}^{l} m_{j} \cdot\left(s_{i_{1}} s_{i_{2}} \cdots s_{i_{j}}\left[i_{j}\right]\right),
$$

is column-equivalent to $D$. In the above, $m \cdot C$ denotes $C \oplus \cdots \oplus C$ with $m$ copies of $C$; in particular $0 \cdot C$ should be interpreted as a diagram with no columns, not the empty column.

The algorithm to produce $\boldsymbol{i}$ and $\boldsymbol{m}$ from $D$ is as follows. To begin the first step, for each $j \in[n]$ let $k_{j}=$ mult $_{D}([j])$, the number of columns of $D$ of the form [ $\left.j\right]$. Replace all such columns by empty columns for each $j$ to get a new diagram $D_{-}$.

Given a column $C \subseteq[n]$, a missing tooth of $C$ is a positive integer $i$ such that $i \notin C$, but $i+1 \in C$. The only columns without missing teeth are the empty column and the intervals [i]. Hence the first nonempty column of $D_{-}$(if there is any) contains a smallest missing tooth $i_{1}$. Switch rows $i_{1}$ and $i_{1}+1$ of $D_{-}$to get a new diagram $D^{\prime}$.

In the second step, repeat the above with $D^{\prime}$ in place of $D$. That is, let $m_{1}=$ mult $_{D^{\prime}}\left(\left[i_{1}\right]\right)$ and replace all columns of the form $\left[i_{1}\right]$ in $D^{\prime}$ by empty columns to get a new diagram $D_{-}^{\prime}$. Find the smallest missing tooth $i_{2}$ of the first nonempty column of $D_{-}^{\prime}$, and switch rows $i_{2}$ and $i_{2}+1$ of $D_{-}^{\prime}$ to get a new diagram $D^{\prime \prime}$.

Continue in this fashion until no nonempty columns remain. It is easily seen that the sequences $\boldsymbol{i}=\left(i_{1}, \ldots, i_{l}\right)$ and $\boldsymbol{m}=\left(k_{1}, \ldots, k_{n} ; m_{1}, \ldots, m_{l}\right)$ just constructed have the desired properties. 
Definition 2.2 The pair $(\boldsymbol{i}, \boldsymbol{m})$ constructed from the preceding algorithm is called the orthodontic sequence of $w$.

Example 2.3 If $w=31542$, then the orthodontic sequence algorithm produces the diagrams
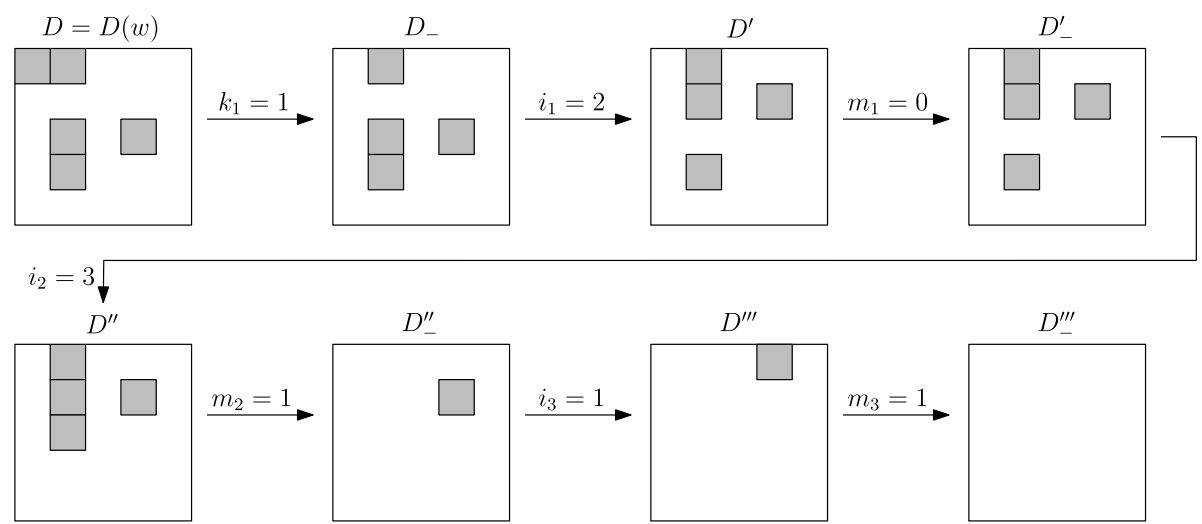

The sequence of missing teeth gives $\boldsymbol{i}=(2,3,1)$ and $\boldsymbol{m}=(1,0,0,0,0 ; 0,1,1)$, so

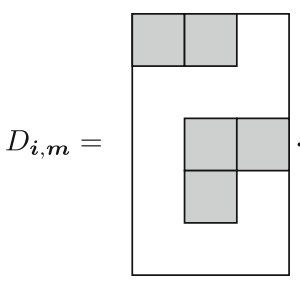

Theorem 2.4 [13, Proposition 15] Let $w \in S_{n}$ have orthodontic sequence $(\boldsymbol{i}, \boldsymbol{m})$. If $\pi_{j}=$ $\partial_{j} x_{j}$ denotes the $j$ th Demazure operator and $\omega_{j}=x_{1} x_{2} \cdots x_{j}$, then

$$
\mathfrak{S}_{w}=\omega_{1}^{k_{1}} \cdots \omega_{n}^{k_{n}} \pi_{i_{1}}\left(\omega_{i_{1}}^{m_{1}} \pi_{i_{2}}\left(\omega_{i_{2}}^{m_{2}} \cdots \pi_{i_{l}}\left(\omega_{i_{l}}^{m_{l}}\right) \cdots\right)\right) .
$$

Example 2.5 For $w=31542$, it is easily checked that

$$
\mathfrak{S}_{w}=x_{1} \pi_{2} \pi_{3}\left(x_{1} x_{2} x_{3} \pi_{1}\left(x_{1}\right)\right) .
$$

Theorem 2.4 can also be realized on the level of tableaux, analogous to Young tableaux in the case of Schur polynomials. A filling (with entries in $\{1, \ldots, n\}$ ) of a diagram $D$ is a map $T$ assigning to each box in $D$ an integer in $[n]$. A filling $T$ is called column-strict if $T$ is strictly increasing down each column of $D$. The weight of a filling $T$ is the vector $w t(T)$ whose $i$ th component $w t(T)_{i}$ is the number of times $i$ occurs in $T$.

Given a permutation $w \in S_{n}$ with orthodontic sequence $(\boldsymbol{i}, \boldsymbol{m})$, we will define a set $\mathcal{T}_{w}$ of fillings of the diagram $D_{i, m}$ which satisfy

$$
\mathfrak{S}_{w}=\sum_{T \in \mathcal{T}_{w}} x_{1}^{w t(T)_{1}} x_{2}^{w t(T)_{2}} \cdots x_{n}^{w t(T)_{n}} .
$$

We start by recalling the root operators, first defined in [11]. These are operators $f_{i}$ which either take a filling $T$ of a diagram $D$ to another filling of $D$, or are undefined on $T$. To define root operators, we first encode a filling $T$ in terms of its reading word. The reading word of 
a filling $T$ of a diagram $D=D_{i, m}$ is the sequence of the entries of $T$ read in order, down each column going left-to-right along columns; that is the sequence

$$
(1,1), T(2,1), \ldots, T(n, 1), T(1,2), T(2,2), \ldots, T(n, 2), \ldots, T(n, n)
$$

ignoring any boxes $(i, j) \notin D$.

If it is defined, the operator $f_{i}$ changes an entry of $i$ in $T$ to an entry of $i+1$ according to the following rule. First, ignore all the entries in $T$ except those which equal $i$ or $i+1$. Now "match parentheses": if, in the list of entries not yet ignored, an $i$ is followed by an $i+1$, then henceforth ignore that pair of entries as well; look again for an $i$ followed (up to ignored entries) by an $i+1$, and henceforth ignore this pair; continue doing this until all no such pairs remain unignored. The remaining entries of $T$ will be a subword of the form $i+1, i+1, \ldots, i+1, i, i, \ldots, i$. If $i$ does not appear in this word, then $f_{i}(T)$ is undefined. Otherwise, $f_{i}$ changes the leftmost $i$ to an $i+1$. Reading the image word back into $D$ produces a new filling. We can iteratively apply $f_{i}$ to a filling $T$.

Example 2.6 If $T=3122213124324131$, applying $f_{1}$ iteratively to $T$ yields:

$$
\begin{aligned}
& T= 3122213124324131 \\
& \cdot 12221 \cdot 12 \cdots 2 \cdot 1 \cdot 1 \\
& \cdots 221 \cdots \cdot 2 \cdot 1 \cdot 1 \\
& \cdots 22 \cdots \cdots \cdot 1 \cdot 1 \\
& f_{1}(T)= 3122213124324231 \\
& f_{1}^{2}(T)= 3122213124324232 \\
& f_{1}^{3}(T) \text { is undefined }
\end{aligned}
$$

Define the set-valued quantized Demazure operator $\tilde{\pi}_{i}$ by $\tilde{\pi}_{i}(T)=\left\{T, f_{i}(t), f_{i}^{2}(T), \ldots\right\}$. For a set $\mathcal{T}$ of tableaux, let

$$
\tilde{\pi}_{i}(\mathcal{T})=\bigcup_{T \in \mathcal{T}} \tilde{\pi}_{i}(T) .
$$

Next, consider the column $[j]$ and its minimal column-strict filling $\widetilde{\omega}_{j}$ ( $j$ th row maps to $j$ ). For a filling $T$ of a diagram $D$ with columns $\left(C_{1}, C_{2}, \ldots, C_{n}\right)$, define in the obvious way the composite filling of $[j] \oplus D$, corresponding to concatenating the reading words of $[j]$ and $D$. Define $[j]^{r} \oplus D$ analogously by adding $r$ columns $[j]$ to $D$, each with filling $\widetilde{\omega}_{j}$.

Definition 2.7 Let $w \in S_{n}$ be a permutation with orthodontic sequence $(\boldsymbol{i}, \boldsymbol{m})$. Define the set $\mathcal{T}_{w}$ of tableaux by

$$
\mathcal{T}_{w}=\widetilde{\omega}_{1}^{k_{1}} \oplus \cdots \oplus \widetilde{\omega}_{n}^{k_{n}} \oplus \tilde{\pi}_{i_{1}}\left(\widetilde{\omega}_{i_{1}}^{m_{1}} \oplus \tilde{\pi}_{i_{2}}\left(\widetilde{\omega}_{i_{2}}^{m_{2}} \oplus \cdots \oplus \tilde{\pi}_{i_{l}}\left(\widetilde{\omega}_{i_{l}}^{m_{l}}\right) \cdots\right)\right) .
$$

Theorem 2.8 [13, Proposition 16] Let $w \in S_{n}$ be a permutation with orthodontic sequence $(\boldsymbol{i}, \boldsymbol{m})$. Then,

$$
\mathfrak{S}_{w}=\sum_{T \in \mathcal{T}_{w}} x_{1}^{w t(T)_{1}} x_{2}^{w t(T)_{2}} \cdots x_{n}^{w t(T)_{n}} .
$$

Example 2.9 Consider again $w=31542$, so the orthodontic sequence of $w$ is $\boldsymbol{i}=(2,3,1)$ and $\boldsymbol{m}=(1,0,0,0,0 ; 0,1,1)$. The set $\mathcal{T}_{w}$ is built up as follows:

$$
\begin{aligned}
\{\} & \stackrel{\widetilde{\omega}_{1}}{\rightarrow}\{1\} \stackrel{\tilde{\pi}_{1}}{\longrightarrow}\{1,2\} \stackrel{\widetilde{\omega}_{3}}{\longrightarrow}\{1231,1232\} \stackrel{\widetilde{\pi}_{3}}{\longrightarrow}\{1231,1241,1232,1242\} \\
& \stackrel{\tilde{\pi}_{2}}{\longrightarrow}\{1231,1241,1341,1232,1233,1242,1342,1343\}
\end{aligned}
$$




\section{$\stackrel{\widetilde{\omega}_{1}}{\longrightarrow}\{11231,11241,11341,11232,11233,11242,11342,11343\}$}

which agrees with

$\mathfrak{S}_{w}=x_{1}^{3} x_{2} x_{3}+x_{1}^{3} x_{2} x_{4}+x_{1}^{3} x_{3} x_{4}+x_{1}^{2} x_{2}^{2} x_{3}+x_{1}^{2} x_{2} x_{3}^{2}+x_{1}^{2} x_{2}^{2} x_{4}+x_{1}^{2} x_{2} x_{3} x_{4}+x_{1}^{2} x_{3}^{2} x_{4}$.

We now describe a way to view each step of the construction of $\mathcal{T}_{w}$ as producing a set of fillings of a diagram.

Definition 2.10 Let $w$ be a permutation with orthodontic sequence $(\boldsymbol{i}, \boldsymbol{m}), \boldsymbol{i}=\left(i_{1}, \ldots, i_{l}\right)$. For each $r \in[l]$, define

$$
\mathcal{T}_{w}(r)=\widetilde{\omega}_{i_{r}}^{m_{r}} \oplus \tilde{\pi}_{i_{r+1}}\left(\widetilde{\omega}_{i_{r+1}}^{m_{r+1}} \oplus \cdots \oplus \tilde{\pi}_{i_{l}}\left(\widetilde{\omega}_{i_{l}}\right) \cdots\right) .
$$

Set $\mathcal{T}_{w}(0)=\mathcal{T}_{w}$

Definition 2.11 Let $w$ be a permutation with orthodontic sequence $(\boldsymbol{i}, \boldsymbol{m}), \boldsymbol{i}=\left(i_{1}, \ldots, i_{l}\right)$. For any $r \in[l]$, let $O(w, r)$ be the diagram obtained from $D(w)$ in the construction of $(\boldsymbol{i}, \boldsymbol{m})$ at the time when the row swaps of the missing teeth $i_{1}, \ldots, i_{r}$ have all been executed on $D(w)$, but after executing the row swap of the missing tooth $i_{r}$, columns without missing teeth have not yet been removed ( $m_{r}$ has not yet been recorded). Set $O(w, 0)=D(w)$. For each $r$, give $O(w, r)$ the same column indexing as $D(w)$, so any columns replaced by empty columns in the execution of the missing teeth $i_{1}, \ldots, i_{r-1}$ retain their original index in $D(w)$.

The motivation behind Definition 2.10 and Definition 2.11 is that the elements of $\mathcal{T}_{w}(r)$ can be viewed as column-strict fillings of $O(w, r)$ for each $r$. To do this, the choice of filling order for $O(w, r)$ is crucial. Let $w \in S_{n}$ and consider $D=D(w)$ and $D_{i, \boldsymbol{m}}$. Suppose $D$ has $z$ nonempty columns. There is a unique permutation $\tau$ of $[n]$ taking the column indices of $D$ to the column indices of $D_{\boldsymbol{i}, \boldsymbol{m}} \oplus \emptyset^{n-z}$ with the following properties:

- Column $c$ of $D$ is the same as column $\tau(c)$ of $D_{i, \boldsymbol{m}}$.

- If column $c$ and column $c^{\prime}$ of $D$ are equal with $c<c^{\prime}$, then $\tau(c)<\tau\left(c^{\prime}\right)$.

Recall that the columns of $O(w, r)$ have the same column labels as $D$. To read an element $T \in$ $\mathcal{T}_{w}(r)$ into $O(w, r)$, read $T$ left-to-right and fill in top-to-bottom columns $\tau^{-1}(n), \tau^{-1}(n-$ $1), \ldots, \tau^{-1}(1)$ (ignoring any column indices referring to empty columns).

Lemma 2.12 Let $w \in S_{n}$ have orthodontic sequence $(\boldsymbol{i}, \boldsymbol{m}), \boldsymbol{i}=\left(i_{1}, \ldots, i_{l}\right)$. In the filling order specified above, the elements of $\mathcal{T}_{w}(r)$ are column-strict fillings of $O(w, r)$ for each $0 \leq r \leq l$.

Example 2.13 Take again $w=31542$ with orthodontic sequence $\boldsymbol{i}=(2,3,1)$ and $\boldsymbol{m}=$ $(1,0,0,0,0 ; 0,1,1)$. Recall that

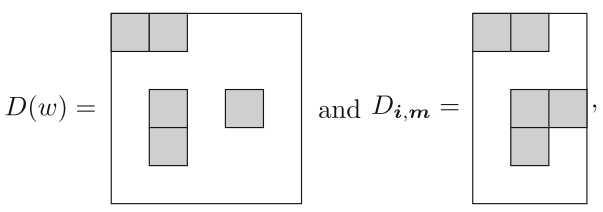

so $\tau=12435=\tau^{-1}$. Consider the elements $1 \in \mathcal{T}_{w}(3), 1232 \in \mathcal{T}_{w}(2), 1242 \in \mathcal{T}_{w}(1)$, and $11342 \in \mathcal{T}_{w}(0)$. The column filling order of each $O(w, r)$ is given by reading $\tau^{-1}$ in one-line notation right to left: in the indexing of $D(w)$, fill down column 4 , then down 
column 2, then down column 1 . The elements of each set $\mathcal{T}_{w}(r)$ are column-strict fillings in the corresponding diagrams $O(w, r)$ :

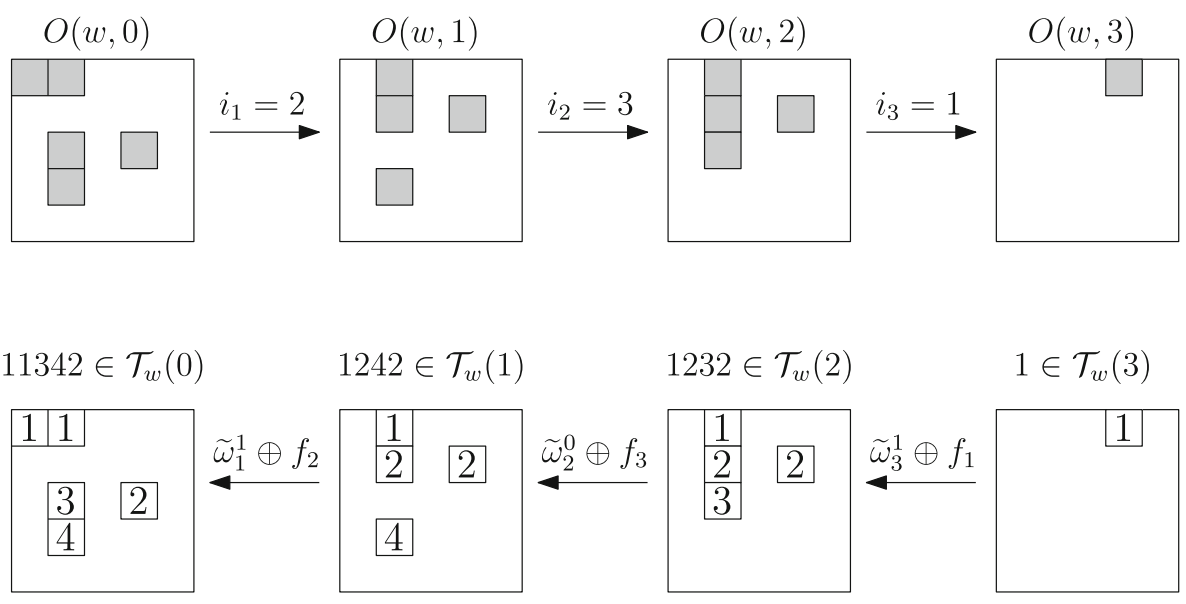

Lemma 2.14 Let $w$ be a permutation with orthodontic sequence $(\boldsymbol{i}, \boldsymbol{m}), \boldsymbol{i}=\left(i_{1}, \ldots, i_{l}\right)$. For each $0 \leq r \leq l, O(w, r)$ has the northwest property.

Definition 2.15 A filling $T$ of a diagram $D$ is called row-flagged if $T(p, q) \leq p$ for each box $(p, q) \in D$.

Lemma 2.16 For each $0 \leq r \leq l$, the elements of $\mathcal{T}_{w}(r)$ are row-flagged fillings of $O(w, r)$.

Proof Clearly, the singleton $\mathcal{T}_{w}(l)$ is a row-flagged filling of $O(w, l)$. Assume that for some $l \geq s>0$, the result holds with $r=s$. We show that the result also holds with $r=s-1$. Let $T \in \mathcal{T}_{w}(s)$. We must show that for each $u$, if $f_{i_{s}}^{u}(T)$ is defined, then $\widetilde{\omega}_{i_{s-1}}^{m_{s-1}} \oplus f_{i_{s}}^{u}(T)$ is a row-flagged filling of $O(w, s-1)$. By the orthodontia construction, $O(w, s)$ is obtained from $O(w, s-1)$ by removing the $m_{s-1}$ columns with no missing tooth, and then switching rows $i_{s}+1$ and $i_{s}$.

Since $T$ is a row-flagged filling of $O(w, s)$, each box in $O(w, s)$ containing an entry of $T$ equal to $i_{s}$ lies in a row with index at least $i_{s}$. Any box in $O(w, s)$ containing an entry of $T$ equal to $i_{s}$ and lying in row $i_{s}$ of $O(w, s)$ will have row index $i_{s}+1$ in $O(w, s-1)$. Any box in $O(w, s)$ containing an entry in $T$ equal to $i_{s}$ and lying in a row $d>i_{s}$ of $O(w, s)$ will still have row index $d$ in $O(w, s-1)$. Then if $f_{i_{s}}^{u}(T)$ is defined, $\widetilde{\omega}_{i_{s-1}}^{m_{s-1}} \oplus f_{i_{s}}^{u}(T)$ will be a row-flagged filling of $O(w, s-1)$.

\section{Zero-one Schubert polynomials}

This section is devoted to giving a sufficient condition on the orthodontic sequence $(\boldsymbol{i}, \boldsymbol{m})$ of $w$ for the Schubert polynomial $\mathfrak{S}_{w}$ to be zero-one. We give such a condition in Theorem 3.6. We will see in Theorem 4.8 that this condition turns out to also be a necessary condition for $\mathfrak{S}_{w}$ to be zero-one.

We start with a less ambitious result:

Proposition 3.1 Let $w \in S_{n}$ and $(\boldsymbol{i}, \boldsymbol{m})$ be the orthodontic sequence of $w$. If $\boldsymbol{i}=\left(i_{1}, \ldots, i_{l}\right)$ has distinct entries, then $\mathfrak{S}_{w}$ is zero-one. 
Proof Let $T, T^{\prime} \in \mathcal{T}_{w}$ with $w t(T)=w t\left(T^{\prime}\right)$. By Definition 2.7, we can find $p_{1}, \ldots, p_{l}$ so that

$$
T=\widetilde{\omega}_{1}^{k_{1}} \oplus \cdots \oplus \widetilde{\omega}_{n}^{k_{n}} \oplus f_{i_{1}}^{p_{1}}\left(\widetilde{\omega}_{i_{1}}^{m_{1}} \oplus \cdots \oplus f_{i_{l}}^{p_{l}}\left(\widetilde{\omega}_{i_{l}}^{m_{l}}\right) \cdots\right) .
$$

Then if $e_{1}, \ldots, e_{n}$ denote the standard basis vectors of $\mathbb{R}^{n}$,

$$
w t(T)=\sum_{j=1}^{n} w t\left(\widetilde{\omega}_{j}^{k_{j}}\right)+\sum_{j=1}^{l} w t\left(\widetilde{\omega}_{i_{j}}^{m_{j}}\right)+\sum_{j=1}^{l} p_{j}\left(e_{i_{j}+1}-e_{i_{j}}\right) .
$$

Similarly, we can find $q_{1}, \ldots, q_{l}$ so that

$$
T^{\prime}=\widetilde{\omega}_{1}^{k_{1}} \oplus \cdots \oplus \widetilde{\omega}_{n}^{k_{n}} \oplus f_{i_{1}}^{q_{1}}\left(\widetilde{\omega}_{i_{1}}^{m_{1}} \oplus \cdots \oplus f_{i_{l}}^{q_{l}}\left(\widetilde{\omega}_{i_{l}}^{m_{l}}\right) \cdots\right),
$$

which implies

$$
w t\left(T^{\prime}\right)=\sum_{j=1}^{n} w t\left(\widetilde{\omega}_{j}^{k_{j}}\right)+\sum_{j=1}^{l} w t\left(\widetilde{\omega}_{i_{j}}^{m_{j}}\right)+\sum_{j=1}^{l} q_{j}\left(e_{i_{j}+1}-e_{i_{j}}\right) .
$$

As $w t(T)=w t\left(T^{\prime}\right)$

$(*) \quad 0=w t(T)-w t\left(T^{\prime}\right)=\left(p_{1}-q_{1}\right)\left(e_{i_{1}+1}-e_{i_{1}}\right)+\cdots+\left(p_{l}-q_{l}\right)\left(e_{i_{l}+1}-e_{i_{l}}\right)$.

Since the vectors $\left\{e_{i_{j}+1}-e_{i_{j}}\right\}_{j=1}^{l}$ are independent and $i$ has distinct entries, $p_{j}=q_{j}$ for all $j$. Thus $T=T^{\prime}$. This shows that all elements of $\mathcal{T}_{w}$ have distinct weights, so $\mathfrak{S}_{w}$ is zero-one.

We now strengthen Proposition 3.1 to allow $\boldsymbol{i}$ to not have distinct entries. To do this, we will need a technical definition related to the orthodontic sequence. Recall the construction of the orthodontic sequence $(\boldsymbol{i}, \boldsymbol{m})$ of a permutation $w \in S_{n}$ (Definition 2.2) and the intermediate diagrams $O(w, r)$ (Definition 2.11). Let $\boldsymbol{i}=\left(i_{1}, \ldots, i_{l}\right)$, and define $O(w, r)_{-}$to be the diagram $O(w, r)$ with all columns of the form $\left[i_{r}\right]$ replaced by empty columns.

Definition 3.2 Define the orthodontic impact function $\mathcal{I}_{w}:[l] \rightarrow 2^{[n]}$ by

$$
\mathcal{I}_{w}(j)=\left\{c \in[n] \mid\left(i_{j}+1, c\right) \in O(w, j-1)_{-}\right\} .
$$

That is, $\mathcal{I}_{w}(j)$ is the set of indices of columns of $O(w, j-1)_{-}$that are changed when rows $i_{j}$ and $i_{j}+1$ are swapped to form $O(w, j)$.

Definition 3.3 Let $w \in S_{n}$ have orthodontic sequence $(\boldsymbol{i}, \boldsymbol{m}), \boldsymbol{i}=\left(i_{1}, \ldots, i_{l}\right)$. We say $w$ is multiplicity-free if for any $r, s \in[l]$ with $r \neq s$ and $i_{r}=i_{s}$, we have $\mathcal{I}_{w}(r)=\mathcal{I}_{w}(s)=\{c\}$ for some $c \in[n]$.

Example 3.4 If $w=457812693$, then

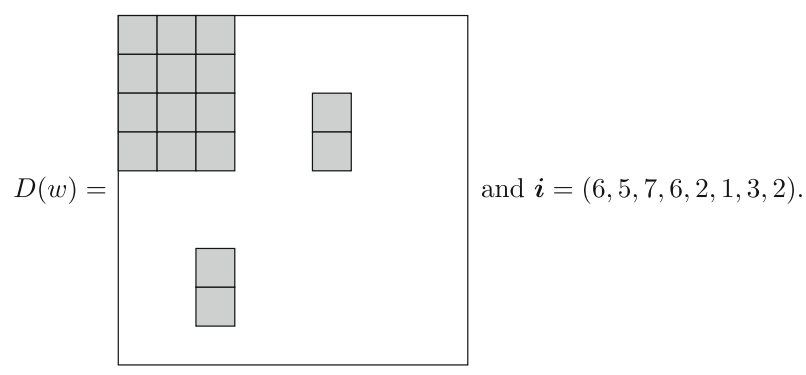


The only entries of $\boldsymbol{i}$ occurring multiple times are $i_{1}=i_{4}=6$ and $i_{5}=i_{8}=2$. Their respective impacts are $\mathcal{I}_{w}(1)=\mathcal{I}_{w}(4)=\{3\}$ and $\mathcal{I}_{w}(5)=\mathcal{I}_{w}(8)=\{6\}$, so $w$ is multiplicityfree.

The proof of the generalization of Proposition 3.1 will require the following technical lemma. Before proceeding, recall Lemma 2.12 and Lemma 2.16: for every $0 \leq j \leq l$, elements of $\mathcal{T}_{w}(j)$ can be viewed as row-flagged, column-strict fillings of $O(w, j)$ (via the column filling order of $O(w, j)$ specified prior to Lemma 2.12). Applying $\widetilde{\omega}_{i_{j-1}}^{m_{j-1}} \oplus f_{i_{j}}$ to an element of $\mathcal{T}_{w}(j)$ gives an element of $\mathcal{T}_{w}(j-1)$, a filling of $O(w, j-1)$. Thus, when we speak below of the application of $f_{i_{j}}$ to an element $T \in \mathcal{T}_{w}(j)$ "changing an $i_{j}$ to an $i_{j}+1$ in column $c$ ", we specifically mean that when we view $T$ as a filling of $O(w, j)$ and $\widetilde{\omega}_{i_{j-1}}^{m_{j-1}} \oplus f_{i_{j}}(T)$ as a filling of $O(w, j-1), T$ and $\widetilde{\omega}_{i_{j-1}}^{m_{j-1}} \oplus f_{i_{j}}(T)$ differ (in the stated way) in their entries in column $c$.

Lemma 3.5 Let $w$ be a multiplicity-free permutation with orthodontic sequence $(\boldsymbol{i}, \boldsymbol{m}), \boldsymbol{i}=$ $\left(i_{1}, \ldots, i_{l}\right)$. Suppose $i_{r}=i_{s}$ with $r<s$ and $\mathcal{I}_{w}(r)=\mathcal{I}_{w}(s)=\{c\}$. Then for each $j$ with $r \leq j \leq s, \mathcal{I}_{w}(j)=\{c\}$ and the application of $f_{i_{j}}$ to an element of $\mathcal{T}_{w}(j)$ is either undefined or changes an $i_{j}$ to an $i_{j}+1$ in column $c$.

Proof We handle first the case that $j=r$. In the diagram $O(w, r-1)$, column $c$ is the leftmost column containing a missing tooth, and $i_{r}$ is the smallest missing tooth in column $c$. Reading column $c$ of $O(w, r-1)$ top-to-bottom, one sees a (possibly empty) sequence of boxes in $O(w, r-1)$, followed by a sequence of boxes not in $O(w, r-1)$. The sequence of boxes not in $O(w, r-1)$ has length at least two since $i_{r}$ occurs at least twice in $\boldsymbol{i}$, and terminates with the box $\left(i_{r}+1, c\right) \in O(w, r-1)$. Note that since $\left(i_{r}-1, c\right),\left(i_{r}, c\right) \notin O(w, r-1)$, the northwest property of $O(w, r-1)$ implies that there can be no box $\left(i_{r}-1, c^{\prime}\right)$ or $\left(i_{r}, c^{\prime}\right)$ in $O(w, r-1)$ with $c^{\prime}>c$. Note also that since $\mathcal{I}_{w}(r)=\{c\}$, we have $\left(i_{r}+1, c^{\prime}\right) \notin O(w, r-1)$ for each $c^{\prime}>c$. Lastly, observe that for any $c^{\prime}>c$ and $d>i_{r}+1$, there can be no box $\left(d, c^{\prime}\right) \in O(w, r-1)$. Otherwise there would be some $t \in[l]$ with $i_{t}=i_{r}$ and $t \neq r$ such that $c^{\prime} \in \mathcal{I}_{w}(t)$, violating that $w$ is multiplicity-free.

As a consequence of the previous observations, the largest row index that a column $c^{\prime}>c$ of $O(w, r-1)$ can contain a box in is $i_{r}-2$. In particular, Lemma 2.16 implies that the application of $f_{i_{r}}$ to an element of $\mathcal{T}_{w}(r)$ either is undefined or changes an $i_{r}$ to an $i_{r}+1$ in column $c$. This concludes the case that $j=r$.

When $j=s$, an entirely analogous argument works. The only significant difference in the observations is that when column $c$ of $O(w, s-1)$ is read top-to-bottom, the (possibly empty) initial sequence of boxes in $O(w, s-1)$ is followed by a sequence of boxes not in $O(w, s-1)$ with length at least 1 , ending with the box $\left(i_{s}+1, c\right)$. Consequently, the largest row index that a column $c^{\prime}>c$ of $O(w, s-1)$ can contain a box in is $i_{s}-1$. In particular, Lemma 2.16 implies that the application of $f_{i_{s}}$ to an element of $\mathcal{T}_{w}(s)$ either is undefined or changes an $i_{s}$ to an $i_{s}+1$ in column $c$. This concludes the case that $j=s$.

Now, let $r<j<s$. Since $\mathcal{I}_{w}(r)=\mathcal{I}_{w}(s)=\{c\}$, we have $c \in \mathcal{I}_{w}(j)$. If $i_{j}$ occurs multiple times in $\boldsymbol{i}$, then multiplicity-freeness of $w$ implies $\mathcal{I}_{w}(j)=\{c\}$. In this case, we can find $j^{\prime} \neq j$ with $i_{j}=i_{j^{\prime}}$ and apply the previous argument (with $r$ and $s$ replaced by $j$ and $j^{\prime}$ ) to conclude that the application of $f_{i_{j}}$ to an element of $\mathcal{T}_{w}(j)$ is either undefined or changes an $i_{j}$ to an $i_{j}+1$ in column $c$.

Thus, we assume $i_{j}$ occurs only once in $i$. Recall that it was shown above that $O(w, r-1)$ has no boxes $\left(d, c^{\prime}\right)$ with $d>i_{r}$ and $c^{\prime}>c$. Read top-to-bottom, let column $c$ of $O(w, r-1)$ have a (possibly empty) initial sequence of boxes ending with a missing box in row $u$, so 
clearly $u \leq i_{r}-1$. Since the first missing tooth in column $c$ of $O(w, r-1)$ is in row $i_{r}$, none of the boxes $(u, c),(u+1, c), \ldots,\left(i_{r}, c\right)$ are in $O(w, r-1)$, but $\left(i_{r}+1, c\right) \in O(w, r-1)$. Then, the northwest property implies that there is no box in $O(w, r-1)$ in any column $c^{\prime}>c$ in any of rows $u, u+1, \ldots, i_{r}$. In particular, the largest row index such that a column $c^{\prime}>c$ of $O(w, r-1)$ can contain a box in is $u-1$.

As $r<j<s$ and $\mathcal{I}_{w}(r)=\mathcal{I}_{w}(s)=\{c\}$, we have that $c \in \mathcal{I}_{w}(j)$. Also since $r<j<s$, the leftmost nonempty column in $O(w, j-1)$ is column $c$, and $i_{j} \geq u$. Then in $O(w, j-1)$, the maximum row index a box in a column $c^{\prime}>c$ can have is $u-1$. In particular, $\mathcal{I}_{w}(j)=\{c\}$, and Lemma 2.16 implies that the application of $f_{i_{j}}$ to an element of $\mathcal{T}_{w}(j)$ is either undefined or changes an $i_{j}$ to an $i_{j}+1$ in column $c$.

Theorem 3.6 If $w$ is multiplicity-free, then $\mathfrak{S}_{w}$ is zero-one.

Proof Assume $w t(T)=w t\left(T^{\prime}\right)$ for some $T, T^{\prime} \in \mathcal{T}_{w}$. If we can show that $T=T^{\prime}$, then we can conclude that all elements of $\mathcal{T}_{w}$ have distinct weights, so $\mathfrak{S}_{w}$ is zero-one. To begin, write

$$
T=\widetilde{\omega}_{1}^{k_{1}} \oplus \cdots \oplus \widetilde{\omega}_{n}^{k_{n}} \oplus f_{i_{1}}^{p_{1}}\left(\widetilde{\omega}_{i_{1}}^{m_{1}} \oplus \cdots \oplus f_{i_{l}}^{p_{l}}\left(\widetilde{\omega}_{i_{l}}^{m_{l}}\right) \cdots\right)
$$

and

$$
T^{\prime}=\widetilde{\omega}_{1}^{k_{1}} \oplus \cdots \oplus \widetilde{\omega}_{n}^{k_{n}} \oplus f_{i_{1}}^{q_{1}}\left(\widetilde{\omega}_{i_{1}}^{m_{1}} \oplus \cdots \oplus f_{i_{l}}^{q_{l}}\left(\widetilde{\omega}_{i_{l}}^{m_{l}}\right) \cdots\right),
$$

for some $p_{1}, \ldots, p_{l}, q_{1}, \ldots, q_{l}$. The basic idea of the proof is to show that as $T$ and $T^{\prime}$ are constructed step-by-step from $\widetilde{\omega}_{i_{l}}^{m_{l}}$, the resulting intermediate tableaux are intermittently equal. At termination of the construction, this will imply that $T=T^{\prime}$.

By the expansion $(*)$ of $w t(T)-w t\left(T^{\prime}\right)$ used in the proof of Proposition 3.1, we observe that $p_{u}=q_{u}$ for all $u$ such that $i_{u}$ occurs only once in $i$. Let $s$ be the largest index such that $p_{s} \neq q_{s}$. Suppose $\mathcal{I}_{w}(s)=\{c\}$. Let $r_{1}$ be the smallest index such that $i_{r_{1}}$ occurs multiple times in $i$ and $\mathcal{I}_{w}\left(r_{1}\right)=\{c\}$. We know $r_{1}<s$, because $(*)$ implies that $p_{s^{\prime}} \neq q_{s^{\prime}}$ for another $s^{\prime}<s$ with $i_{s^{\prime}}=i_{s}$, and by multiplicity-freeness $\mathcal{I}_{w}\left(s^{\prime}\right)=\{c\}$. We wish to find an interval $[r, s] \subseteq\left[r_{1}, s\right]$ such that $r<s$ and the following two conditions hold:

(i) If $v \geq r$ and $i_{v}$ occurs multiple times in $i$, then any other $v^{\prime}$ with $i_{v}=i_{v^{\prime}}$ will satisfy $v^{\prime} \geq r$.

(ii) For every $j$ with $r<j<s$ and $i_{j}$ occurring only once in $i$, there are $t$ and $u$ with $r \leq t<j<u \leq s$ such that $i_{t}=i_{u}$.

We first show that (i) holds for $\left[r_{1}, s\right]$. Note that if $i_{v}$ occurs multiple times in $i$ and $r_{1} \leq v \leq s$, then it must be that $\mathcal{I}_{w}(v)=\{c\}$ by the fact that the orthodontia construction records all missing teeth needed to eliminate one column before moving on to the next column. If $i_{v^{\prime}}=i_{v}$, then $\mathcal{I}_{w}\left(v^{\prime}\right)=\{c\}$ also, by multiplicity-freeness of $w$. The choice of $r_{1}$ implies $r_{1} \leq v^{\prime}$. If $i_{v}$ occurs multiple times in $i$ with $s<v$ and $\mathcal{I}_{w}(v)=\{c\}$, then the choice of $r_{1}$ again implies that $r_{1} \leq v^{\prime}$ for any $i_{v^{\prime}}=i_{v}$. If $i_{v}$ occurs multiple times in $i$ with $s<v$ and $\mathcal{I}_{w}(v) \neq\{c\}$, then the orthodontia construction implies that any $v^{\prime}$ with $i_{v}=i_{v^{\prime}}$ must satisfy $s<v^{\prime}$. In particular, $r_{1}<v^{\prime}$ as needed. Thus, (i) holds for $\left[r_{1}, s\right]$. If $\left[r_{1}, s\right]$ also satisfies (ii), then we are done.

Otherwise, assume $\left[r_{1}, s\right]$ does not satisfy (ii). Then there is some $j$ with $r_{1}<j<s$ such that $i_{j}$ occurs only once in $i$ and there are no $t$ and $u$ with $r_{1} \leq t<j<u \leq s$ and $i_{t}=i_{u}$. Consequently for every pair $i_{u}=i_{t}$ with $r_{1} \leq t<u \leq s$, it must be that either $t<u<j$ or $j<t<u$. Let $r_{2}$ be the smallest index such that $j<r_{2}$ and $i_{r_{2}}$ occurs multiple times in $i$. 
By the choice of $j$, it is clear that the interval $\left[r_{2}, s\right]$ still satisfies (i). If $\left[r_{2}, s\right]$ also satisfies (ii), then we are done.

Otherwise, $\left[r_{2}, s\right]$ satisfies (i) but not (ii), and we can argue exactly as in the case of $\left[r_{1}, s\right]$ to find an $r_{3}$ such that $r_{2}<r_{3}<s$ and $\left[r_{3}, s\right]$ satisfies (i). Continue working in this fashion. We show that this process terminates with an interval $[r, s]$ satisfying $r<s$, (i), and (ii).

As mentioned above, there exists $s^{\prime}<s$ such that $i_{s^{\prime}}=i_{s}$. Let $s^{\prime}$ be the maximal index less than $s$ with this property. Since all of the intervals $\left[r_{*}, s\right]$ will satisfy (i), it follows that $r_{1}<r_{2}<\cdots \leq s^{\prime}$. At worst, the process will terminate after finitely many steps with the interval $\left[s^{\prime}, s\right]$. The interval $\left[s^{\prime}, s\right]$ will then satisfy (i) since the process reached it, and will trivially satisfy (ii) since $i_{s}=i_{s^{\prime}}$.

Hence, we can assume that we have found an interval $[r, s]$ satisfying $r<s$, (i), and (ii). Consider the tableaux

$$
\begin{array}{ll}
T_{r}=\widetilde{\omega}_{i_{r-1}}^{m_{r-1}} \oplus f_{i_{r}}^{p_{r}}\left(\widetilde{\omega}_{i_{r}}^{m_{r}} \oplus \cdots \oplus f_{i_{l}}^{p_{l}}\left(\widetilde{\omega}_{i_{l}}^{m_{l}}\right) \cdots\right), & T_{s}=\widetilde{\omega}_{i_{s}}^{m_{s}} \oplus f_{i_{s+1}}^{p_{s+1}}\left(\widetilde{\omega}_{i_{s+1}}^{m_{s+1}} \oplus \cdots \oplus f_{i_{l}}^{p_{l}}\left(\widetilde{\omega}_{i_{l}}^{m_{l}}\right) \cdots\right), \\
T_{r}^{\prime}=\widetilde{\omega}_{i_{r-1}}^{m_{r-1}} \oplus f_{i_{r}}^{q_{r}}\left(\widetilde{\omega}_{i_{r}}^{m_{r}} \oplus \cdots \oplus f_{i_{l}}^{q_{l}}\left(\widetilde{\omega}_{i_{l}}^{m_{l}}\right) \cdots\right), & T_{s}^{\prime}=\widetilde{\omega}_{i_{s}}^{m_{s}} \oplus f_{i_{s+1}}^{q_{s+1}}\left(\widetilde{\omega}_{i_{s+1}}^{m_{s+1}} \oplus \cdots \oplus f_{i_{l}}^{q_{l}}\left(\widetilde{\omega}_{i_{l}}^{m_{l}}\right) \cdots\right) .
\end{array}
$$

By definition, $T_{r}, T_{r}^{\prime} \in \mathcal{T}_{w}(r-1)$, so we can view $T_{r}$ and $T_{r}^{\prime}$ as fillings of $O(w, r-1)$. Similarly, $T_{s}, T_{s}^{\prime} \in \mathcal{T}_{w}(s)$, so we can view $T_{s}$ and $T_{s}^{\prime}$ as fillings of $O(w, s)$. Since we chose $s$ to be the largest index such that $p_{s} \neq q_{s}$, it follows that $T_{s}=T_{s}^{\prime}$. By property (i) of $[r, s]$, $i_{u} \neq i_{v}$ for any $u<r \leq v$. Hence, it must be that $w t\left(T_{r}\right)=w t\left(T_{r}^{\prime}\right)$. Finally, property (ii) of $[r, s]$ allows us to apply Lemma 3.5 and conclude that for any $a_{r}, a_{r+1}, \ldots, a_{s} \geq 0$, when $\widetilde{\omega}_{i_{r-1}}^{m_{r-1}} \oplus f_{i_{r}}^{a_{r}}\left(\widetilde{\omega}_{m_{r}}^{i_{r}} \oplus \cdots \oplus \widetilde{\omega}_{i_{s-1}}^{m_{s-1}} f_{i_{s}}^{a_{s}}(-) \cdots\right)$ is applied to an element of $\mathcal{T}_{w}(s)$, only the entries in column $c$ are affected by the root operators $f_{i_{r}}^{a_{r}}, \ldots, f_{i_{s}}^{a_{s}}$. Since

$$
\begin{aligned}
T_{r}=\widetilde{\omega}_{i_{r-1}}^{m_{r-1}} \oplus f_{i_{r}}^{p_{r}}\left(\widetilde{\omega}_{m_{r}}^{i_{r}} \oplus \cdots \oplus \widetilde{\omega}_{i_{s-1}}^{m_{s-1}} f_{i_{s}}^{p_{s}}\left(T_{s}\right) \cdots\right) \\
\quad \text { and } T_{r}^{\prime}=\widetilde{\omega}_{i_{r-1}}^{m_{r-1}} \oplus f_{i_{r}}^{q_{r}}\left(\widetilde{\omega}_{m_{r}}^{i_{r}} \oplus \cdots \oplus \widetilde{\omega}_{i_{s-1}}^{m_{s-1}} f_{i_{s}}^{q_{s}}\left(T_{s}^{\prime}\right) \cdots\right),
\end{aligned}
$$

$T_{r}$ and $T_{r}^{\prime}$ must coincide outside of column $c$. Since we already deduced that $w t\left(T_{r}\right)=$ $w t\left(T_{r}^{\prime}\right)$, it follows that column $c$ of $T_{r}$ and $T_{r}^{\prime}$ have the same weight. By column-strictness of $T_{r}$ and $T_{r}^{\prime}$, column $c$ of $T_{r}$ and $T_{r}^{\prime}$ must coincide, so $T_{r}=T_{r}^{\prime}$.

To complete the proof, let $\hat{s}$ be the largest index $\hat{s}<r$ such that $p_{\hat{s}} \neq q_{\hat{s}}$. If no such index exists, then $T=T^{\prime}$. Otherwise, set $\hat{r}_{1}$ to be the smallest index such that $i_{\hat{r}_{1}}$ occurs multiple times in $\boldsymbol{i}$ and $\mathcal{I}_{w}\left(\hat{r}_{1}\right)=\mathcal{I}_{w}(\hat{s})$. We have $\hat{r}_{1}<\hat{s}$ because some other $\hat{s}^{\prime}$ distinct from $\hat{s}$ such that $p_{\hat{s}^{\prime}} \neq q_{\hat{s}^{\prime}}$ and $i_{\hat{s}^{\prime}}=i_{\hat{s}}$ must exist as before, and $\hat{s}^{\prime}$ is also less than $r$ by property (i) of $[r, s]$. Use the previous algorithm to find an interval $[\hat{r}, \hat{s}] \subseteq\left[\hat{r}_{1}, \hat{s}\right]$ satisfying $\hat{r}<\hat{s}$, (i), and (ii). Construct $T_{\hat{r}}, T_{\hat{r}}^{\prime}, T_{\hat{s}}, T_{\hat{s}}^{\prime}$, and argue exactly as in the case of $[r, s]$ that $T_{\hat{r}}=T_{\hat{r}}^{\prime}$.

Continuing in this manner for a finite number of steps will show that $T=T^{\prime}$.

As we will show in Theorem 4.8, it is not only sufficient but also necessary that $w$ be multiplicity-free for the Schubert polynomial $\mathfrak{S}_{w}$ to be zero-one.

\section{Pattern avoidance conditions for multiplicity-freeness}

This section is devoted to showing that $w$ being multiplicity-free is equivalent to a certain pattern avoidance condition. We then prove our full characterization of zero-one Schubert polynomials.

We start with several definitions. 


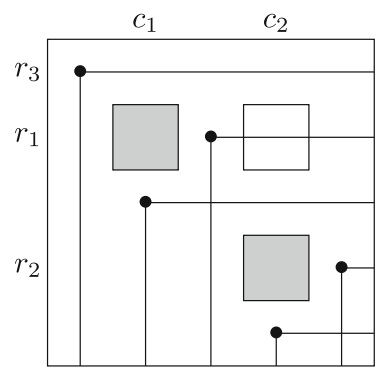

A

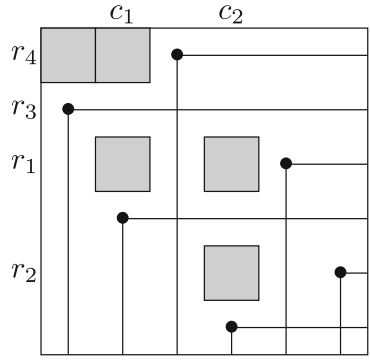

$\mathrm{B}$

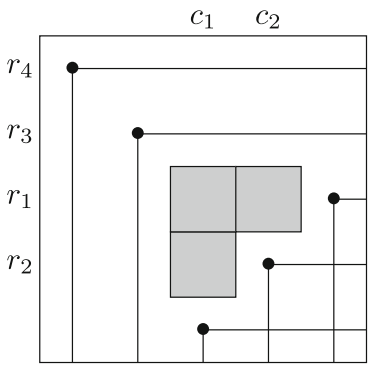

$\mathrm{B}^{\prime}$

Fig. 1 Examples of instances of the configurations A, B, and $\mathrm{B}^{\prime}$ in Rothe diagrams. Both the hooks removed from the $n \times n$ grid to form each Rothe diagram and the remaining boxes are shown

Definition 4.1 We say a Rothe diagram $D=D(w)$ contains an instance of configuration A if there are $r_{1}, c_{1}, r_{2}, c_{2}, r_{3}$ such that $1 \leq r_{3}<r_{1}<r_{2}, 1<c_{1}<c_{2},\left(r_{1}, c_{1}\right),\left(r_{2}, c_{2}\right) \in D$, $\left(r_{1}, c_{2}\right) \notin D$, and $w_{r_{3}}<c_{1}$.

Definition 4.2 We say a Rothe diagram $D=D(w)$ contains an instance of configuration $\mathrm{B}$ if there are $r_{1}, c_{1}, r_{2}, c_{2}, r_{3}, r_{4}$ such that $1 \leq r_{4} \neq r_{3}<r_{1}<r_{2}, 2<c_{1}<c_{2}$, $\left(r_{1}, c_{1}\right),\left(r_{1}, c_{2}\right),\left(r_{2}, c_{2}\right) \in D, w_{r_{3}}<c_{1}$, and $w_{r_{4}}<c_{2}$.

Definition 4.3 We say a Rothe diagram $D=D(w)$ contains an instance of configuration $\mathrm{B}^{\prime}$ if there are $r_{1}, c_{1}, r_{2}, c_{2}, r_{3}, r_{4}$ such that $1 \leq r_{4}<r_{3}<r_{1}<r_{2}, 2<c_{1}<c_{2}$, $\left(r_{1}, c_{1}\right),\left(r_{1}, c_{2}\right),\left(r_{2}, c_{1}\right) \in D, w_{r_{3}}<c_{1}$, and $w_{r_{4}}<c_{1}$.

Given a Rothe diagram $D(w)$, we will call a tuple $\left(r_{1}, c_{1}, r_{2}, c_{2}, r_{3}\right)$ meeting the conditions of Definition 4.1 an instance of configuration A in $D(w)$. Similarly, we will call a tuple $\left(r_{1}, c_{1}, r_{2}, c_{2}, r_{3}, r_{4}\right)$ meeting the conditions of Definition 4.2 (resp. 4.3) an instance of configuration B (resp. $\left.\mathrm{B}^{\prime}\right)$ in $D(w)$ (Fig. 1).

Theorem 4.4 If $w \in S_{n}$ is a permutation such that $D(w)$ does not contain any instance of configuration $A, B$, or $B^{\prime}$, then $w$ is multiplicity-free.

Theorem 4.8 will also imply the converse of this theorem.

Proof We prove the contrapositive. Assume $w$ is not multiplicity-free and let $(\boldsymbol{i}, \boldsymbol{m})$ be the orthodontic sequence of $w$. Then, we can find entries $i_{p_{1}}=i_{p_{2}}$ of $i$ with $p_{1}<p_{2}$ such that either $\mathcal{I}_{w}\left(p_{1}\right) \neq \mathcal{I}_{w}\left(p_{2}\right)$, or $\mathcal{I}_{w}\left(p_{1}\right)=\mathcal{I}_{w}\left(p_{2}\right)$ with $\left|\mathcal{I}_{w}\left(p_{1}\right)\right|>1$. We show that $D(w)$ must contain at least one instance of configuration $\mathrm{A}, \mathrm{B}$, or $\mathrm{B}^{\prime}$.

Case 1: Assume that $\mathcal{I}_{w}\left(p_{1}\right) \nsubseteq \mathcal{I}_{w}\left(p_{2}\right)$ and $\mathcal{I}_{w}\left(p_{2}\right) \nsubseteq \mathcal{I}_{w}\left(p_{1}\right)$. Take $c_{1} \in \mathcal{I}_{w}\left(p_{1}\right) \backslash \mathcal{I}_{w}\left(p_{2}\right)$ and $c_{2} \in \mathcal{I}_{w}\left(p_{2}\right) \backslash \mathcal{I}_{w}\left(p_{1}\right)$. We show that columns $c_{1}$ and $c_{2}$ of $D(w)$ contain an instance of configuration A.

In step $p_{1}$ of the orthodontia on $D(w)$, a box in column $c_{1}$ is moved (by the missing tooth $i_{p_{1}}$ ) to row $i_{p_{1}}$. Let this box originally be in row $r_{1}$ of $D(w)$. Analogously, let the box in column $c_{2}$ moved to row $i_{p_{2}}$ in step $p_{2}$ of the orthodontia (by the missing tooth $i_{p_{2}}$ ) originally be in row $r_{2}$ of $D(w)$. Observe that $r_{1}<r_{2}$. If $c_{2}<c_{1}$, then the northwest property would imply that $\left(r_{1}, c_{2}\right) \in D(w)$, contradicting that $c_{2} \notin \mathcal{I}_{w}\left(p_{1}\right)$. Thus $c_{1}<c_{2}$. Since $c_{2} \notin \mathcal{I}_{w}\left(p_{1}\right)$, $\left(r_{1}, c_{2}\right) \notin D(w)$. Lastly, since the box $\left(r_{1}, c_{1}\right)$ is moved by the orthodontia, there is some box $\left(r_{3}, c_{1}\right) \notin D(w)$ with $r_{3}<r_{1}$. Consequently, $w_{r_{3}}<c_{1}$. Thus, $\left(r_{1}, c_{1}, r_{2}, c_{2}, r_{3}\right)$ is an instance of configuration A. 


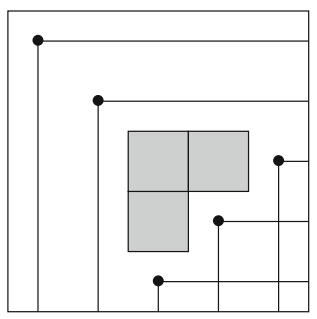

$D(12543)$

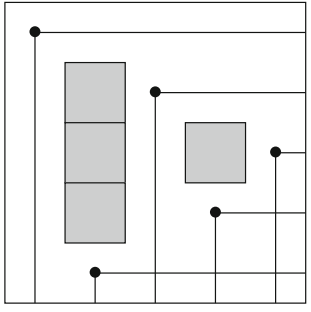

$D(13542)$

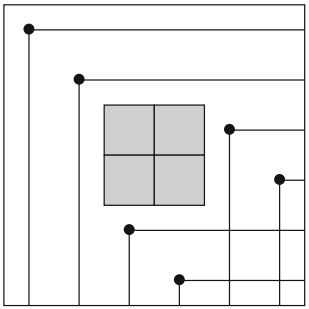

$D(125634)$

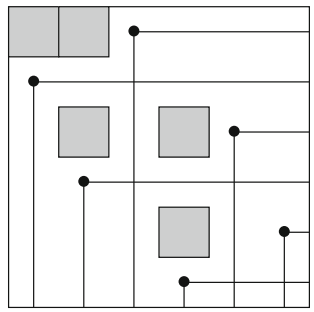

$D(315264)$

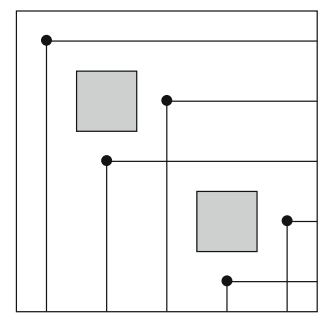

$D(13254)$

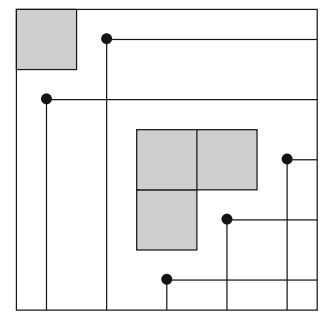

$D(21543)$

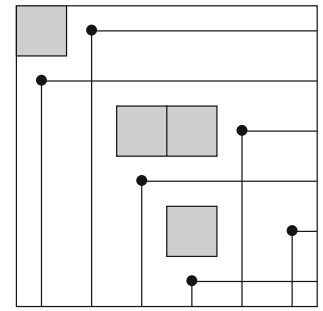

$D(215364)$

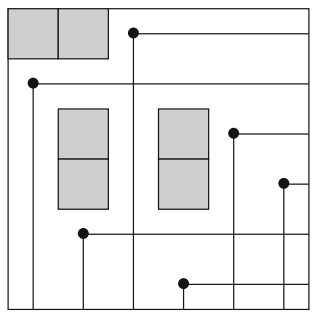

$D(315624)$

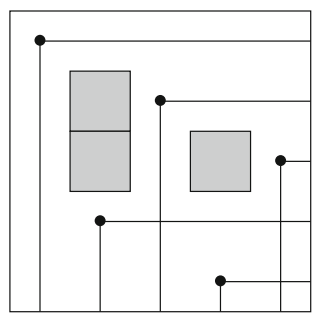

$D(13524)$

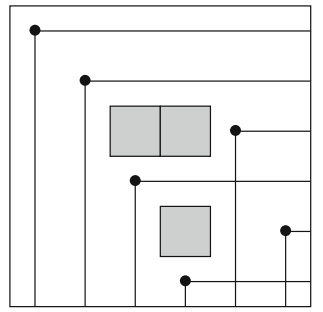

$D(125364)$

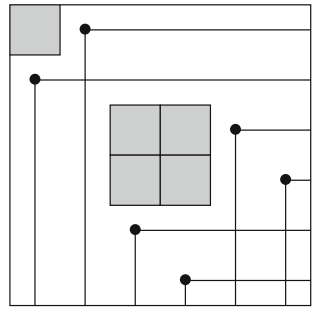

$D(215634)$

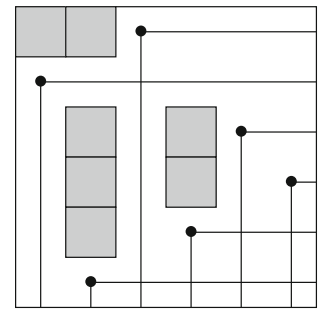

$D(315642)$

Fig. 2 The Rothe diagrams of the twelve multiplicitous patterns

Case 2: Assume $\mathcal{I}_{w}\left(p_{2}\right)$ is a proper subset of $\mathcal{I}_{w}\left(p_{1}\right)$. Let $c_{1}=\max \left(\mathcal{I}_{w}\left(p_{2}\right)\right)$ and $c_{2}=$ $\min \left(\mathcal{I}_{w}\left(p_{1}\right) \backslash \mathcal{I}_{w}\left(p_{2}\right)\right)$. Let the box in column $c_{1}$ moved to row $i_{p_{1}}=i_{p_{2}}$ in step $p_{1}$ (resp. $p_{2}$ ) of the orthodontia originally be in row $r_{1}$ (resp. $r_{2}$ ) of $D(w)$. Observe that $r_{1}<r_{2}$.

Assume first that $c_{1}<c_{2}$. Since $c_{1} \in \mathcal{I}_{w}\left(p_{1}\right) \cap \mathcal{I}_{w}\left(p_{2}\right)$, the boxes $\left(r_{1}, c_{1}\right)$ and $\left(r_{2}, c_{2}\right)$ both move weakly above row $i_{p_{1}}$ in the orthodontia. Then, we can find indices $r_{3}, r_{4}$ with 
$r_{4}<r_{3}<r_{1}$ such that $\left(r_{3}, c_{1}\right),\left(r_{4}, c_{1}\right) \notin D(w)$. Hence, $w_{r_{3}}<c_{1}$ and $w_{r_{4}}<c_{1}$, so $\left(r_{1}, c_{1}, r_{2}, c_{2}, r_{3}, r_{4}\right)$ is an instance of configuration $\mathrm{B}^{\prime}$.

Otherwise $c_{1}>c_{2}$. Since the box $\left(r_{1}, c_{2}\right)$ is moved by the orthodontia, we can find $r_{3}<r_{1}$ with $\left(r_{3}, c_{2}\right) \notin D(w)$. Then $w_{r_{3}}<c_{2}$. As we are assuming $c_{2}<c_{1},\left(r_{3}, c_{1}\right) \notin D(w)$ also. Since the boxes $\left(r_{1}, c_{1}\right)$ and $\left(r_{2}, c_{1}\right)$ in $D(w)$ are moved weakly above row $i_{p_{1}}$ by the orthodontia, we can find some $r_{4}<r_{1}$ with $r_{4} \neq r_{3}$ such that $\left(r_{4}, c_{1}\right) \notin D(w)$. Then, $w_{r_{4}}<c_{1}$, so $\left(r_{1}, c_{2}, r_{2}, c_{1}, r_{3}, r_{4}\right)$ is an instance of configuration $\mathrm{B}$.

Case 3: Assume $\mathcal{I}_{w}\left(p_{1}\right)$ is a proper subset of $\mathcal{I}_{w}\left(p_{2}\right)$. This case is handled similarly to Case 2. Let $c_{1}=\max \left(\mathcal{I}_{w}\left(p_{1}\right)\right)$ and $c_{2}=\min \left(\mathcal{I}_{w}\left(p_{2}\right) \backslash \mathcal{I}_{w}\left(p_{1}\right)\right)$. Let the box in column $c_{1}$ moved to row $i_{p_{1}}=i_{p_{2}}$ in step $p_{1}$ (resp. $p_{2}$ ) of the orthodontia originally be in row $r_{1}$ (resp. $\left.r_{2}\right)$ of $D(w)$. Observe that $r_{1}<r_{2}$.

Assume $c_{1}<c_{2}$. Since the boxes $\left(r_{1}, c_{1}\right)$ and $\left(r_{2}, c_{1}\right)$ of $D(w)$ are moved weakly above row $i_{p_{1}}$ by the orthodontia, we can find indices $r_{3}, r_{4}$ with $r_{4}<r_{3}<r_{1}$ such that $\left(r_{3}, c_{1}\right),\left(r_{4}, c_{1}\right) \notin D(w)$. Then, $w_{r_{3}}<c_{1}$ and $w_{r_{4}}<c_{1}$. Since $c_{2} \notin \mathcal{I}_{w}\left(p_{1}\right)$, $\left(r_{1}, c_{2}\right) \notin D(w)$. Then, $\left(r_{1}, c_{1}, r_{2}, c_{2}, r_{3}\right)$ is an instance of configuration A.

Otherwise $c_{1}>c_{2}$. As $c_{2} \notin \mathcal{I}_{w}\left(p_{1}\right),\left(r_{1}, c_{2}\right) \notin D(w)$. Since $\left(r_{2}, c_{2}\right),\left(r_{1}, c_{1}\right) \in D(w)$, this is a contradiction of the northwest property of $D(w)$.

Case 4: Assume $\mathcal{I}_{w}\left(p_{1}\right)=\mathcal{I}_{w}\left(p_{2}\right)$ is not a singleton. Let $c_{1}, c_{2} \in \mathcal{I}_{w}\left(p_{1}\right)$ with $c_{1}<c_{2}$. Let the box in column $c_{1}$ moved to row $i_{p_{1}}=i_{p_{2}}$ in step $p_{1}$ (resp. $p_{2}$ ) of the orthodontia originally be in row $r_{1}$ (resp. $r_{2}$ ) of $D(w)$. Observe that $r_{1}<r_{2}$. Since the boxes $\left(r_{1}, c_{1}\right)$ and $\left(r_{2}, c_{1}\right)$ in $D(w)$ are moved weakly above row $i_{p_{1}}$ by the orthodontia, we can find indices $r_{3}, r_{4}$ with $r_{4}<r_{3}<r_{1}$ such that $\left(r_{3}, c_{1}\right),\left(r_{4}, c_{1}\right) \notin D(w)$. Then, $w_{r_{3}}<c_{1}$ and $w_{r_{4}}<c_{1}$. Thus, $\left(r_{1}, c_{1}, r_{2}, c_{2}, r_{3}, r_{4}\right)$ is an instance of configuration $\mathrm{B}^{\prime}$.

We now relate multiplicity-freeness to pattern avoidance of permutations. We begin by clarifying our pattern avoidance terminology. A pattern $\sigma$ of length $n$ is a permutation in $S_{n}$. The length $n$ is a crucial part of the data of a pattern; we make no identifications between patterns of different lengths, unlike what is usual when handling permutations in the Schubert calculus. A permutation $w$ contains $\sigma$ if $w$ has $n$ entries $w_{j_{1}}, \ldots, w_{j_{n}}$ with $j_{1}<j_{2}<\cdots<j_{n}$ that are in the same relative order as $\sigma_{1}, \sigma_{2}, \ldots, \sigma_{n}$. In this case, the indices $j_{1}<j_{2}<\cdots<j_{n}$ are called a realization of $\sigma$ in $w$. We say that $w$ avoids the pattern $\sigma$ if $w$ does not contain $\sigma$. To illustrate the dependence of these definitions on $n$, note that $w=154623$ contains the pattern 132, but not the pattern 132456 .

The following easy lemma gives a diagrammatic interpretation of pattern avoidance.

Lemma 4.5 Let $w \in S_{n}$ be a permutation and $\sigma$ a pattern of length $m$ contained in $w$. Choose a realization $j_{1}<j_{2}<\cdots<j_{m}$ of $\sigma$ in $w$. Then $D(\sigma)$ is obtained from $D(w)$ by deleting the rows $[n] \backslash\left\{j_{1}, \ldots, j_{m}\right\}$ and the columns $[n] \backslash\left\{w_{j_{1}}, \ldots, w_{j_{m}}\right\}$, and reindexing the remaining rows and columns by $[\mathrm{m}]$, preserving their order.

Definition 4.6 The multiplicitous patterns are those in the set

MPatt $=\{12543,13254,13524,13542,21543,125364,125634,215364,215634$, $315264,315624,315642\}$.

Theorem 4.7 Let $w \in S_{n}$. Then $D(w)$ does not contain any instance of configuration $A, B$, or $B^{\prime}$ if and only if $w$ avoids all of the multiplicitous patterns.

Proof It is easy to check (see Fig. 2) that each of the twelve multiplicitous patterns contains an instance of configuration $\mathrm{A}, \mathrm{B}$, or $\mathrm{B}^{\prime}$. Lemma 4.5 implies that if $w$ contains $\sigma \in \mathrm{MPatt}$, 
then deleting some rows and columns from $D(w)$ yields $D(\sigma)$. Since $D(\sigma)$ contains at least one instance of configuration $\mathrm{A}, \mathrm{B}$, or $\mathrm{B}^{\prime}$, so does $D(w)$.

Conversely, assume $D(w)$ contains at least one instance of configuration $\mathrm{A}, \mathrm{B}$, or $\mathrm{B}^{\prime}$. We must show that $w$ contains some multiplicitous pattern. Let $\tau^{1}, \tau^{2}, \ldots, \tau^{n}$ be the $n$ patterns of length $n-1$ contained in $w$; say $\tau^{j}$ is realized in $w$ by forgetting $w_{j}$. Without loss of generality, we may assume none of $D\left(\tau^{1}\right), \ldots, D\left(\tau^{n}\right)$ contain an instance of configuration $\mathrm{A}, \mathrm{B}$, or $\mathrm{B}^{\prime}$ : if $D\left(\tau^{j}\right)$ does contain an instance of one of these configurations, replace $w$ by $\tau^{j}$ and iterate.

For each $j, D\left(\tau^{j}\right)$ is obtained from $D(w)$ by deleting row $j$ and column $w_{j}$. Since $D\left(\tau^{j}\right)$ does not contain any instance of any of our three configurations, each cross $\{(j, q) \mid$ $(j, q) \in D(w)\} \cup\left\{\left(p, w_{j}\right) \mid\left(p, w_{j}\right) \in D(w)\right\}$ intersects each instance of every configuration contained in $D(w)$. However, an instance of configuration A involves only three rows and two columns, and an instance of B or B' involves only four rows and two columns. Thus, it must be that $w \in S_{n}$ for some $n \leq 6$. It can be checked by exhaustion that the only permutations in $S_{n}$ with $n \leq 6$ that are minimal (with respect to pattern avoidance) among those whose Rothe diagrams contain an instance of configuration $\mathrm{A}, \mathrm{B}$, or $\mathrm{B}^{\prime}$ are the twelve multiplicitous patterns.

We are now ready to state our full characterization of zero-one Schubert polynomials, and most of the elements of the proof are at hand.

Theorem 4.8 The following are equivalent:

(i) The Schubert polynomial $\mathfrak{S}_{w}$ is zero-one.

(ii) The permutation $w$ is multiplicity-free,

(iii) The Rothe diagram $D(w)$ does not contain any instance of configuration $A, B$, or $B^{\prime}$,

(iv) The permutation $w$ avoids the multiplicitous patterns, namely 12543, 13254, 13524, 13542, 21543, 125364, 125634, 215364, 215634, 315264, 315624, and 315642.

Proof Theorem 3.6 shows $(i i) \Rightarrow(i)$. Theorem 4.4 shows $(i i i) \Rightarrow$ (ii). Theorem 4.7 shows (iii) $\Leftrightarrow(i v)$. The implication $(i) \Rightarrow(i v)$ will follow immediately from Corollary 5.9, since the Schubert polynomials associated to the permutations 12543, 13254, 13524, 13542, 21543, $125364,125634,215364,215634,315264,315624$, and 315642 each have a coefficient equal to 2 . We prove Corollary 5.9 in the next section.

\section{A coefficient-wise inequality for dual characters of flagged Weyl modules of diagrams}

The aim of this section is to prove a generalization of Theorem 1.2, namely, Theorem 5.8. We now explain the necessary background and terminology for Theorem 5.8 and its proof.

Let $G=\operatorname{GL}(n, \mathbb{C})$ be the group of $n \times n$ invertible matrices over $\mathbb{C}$ and $B$ be the subgroup of $G$ consisting of the $n \times n$ upper-triangular matrices. The flagged Weyl module is a representation $\mathcal{M}_{D}$ of $B$ associated to a diagram $D$. The dual character of $\mathcal{M}_{D}$ has been shown in certain cases to be a Schubert polynomial [8] or a key polynomial [16]. We will use the construction of $\mathcal{M}_{D}$ in terms of determinants given in [13].

Denote by $Y$ the $n \times n$ matrix with indeterminates $y_{i j}$ in the upper-triangular positions $i \leq j$ and zeros elsewhere. Let $\mathbb{C}[Y]$ be the polynomial ring in the indeterminates $\left\{y_{i j}\right\}_{i \leq j}$. Note that $B$ acts on $\mathbb{C}[Y]$ on the right via left translation: if $f(Y) \in \mathbb{C}[Y]$, then a matrix $b \in B$ acts on $f$ by $f(Y) \cdot b=f\left(b^{-1} Y\right)$. For any $R, S \subseteq[n]$, let $Y_{S}^{R}$ be the submatrix of $Y$ obtained by restricting to rows $R$ and columns $S$. 
For $R, S \subseteq[n]$, we say $R \leq S$ if \#R=\#S and the $k$ th least element of $R$ does not exceed the $k$ th least element of $S$ for each $k$. For any diagrams $C=\left(C_{1}, \ldots, C_{n}\right)$ and $D=\left(D_{1}, \ldots, D_{n}\right)$, we say $C \leq D$ if $C_{j} \leq D_{j}$ for all $j \in[n]$.

Definition 5.1 For a diagram $D=\left(D_{1}, \ldots, D_{n}\right)$, the flagged Weyl module $\mathcal{M}_{D}$ is defined by

$$
\mathcal{M}_{D}=\operatorname{Span}_{\mathbb{C}}\left\{\prod_{j=1}^{n} \operatorname{det}\left(Y_{D_{j}}^{C_{j}}\right) \mid C \leq D\right\} .
$$

$\mathcal{M}_{D}$ is a $B$-module with the action inherited from the action of $B$ on $\mathbb{C}[Y]$.

Note that since $Y$ is upper-triangular, the condition $C \leq D$ is technically unnecessary since $\operatorname{det}\left(Y_{D_{j}}^{C_{j}}\right)=0$ unless $C_{j} \leq D_{j}$. Conversely, if $C_{j} \leq D_{j}$, then $\operatorname{det}\left(Y_{D_{j}}^{C_{j}}\right) \neq 0$.

For any $B$-module $N$, the character of $N$ is defined by $\operatorname{char}(N)\left(x_{1}, \ldots, x_{n}\right)=$ $\operatorname{tr}(X: N \rightarrow N)$ where $X$ is the diagonal matrix $\operatorname{diag}\left(x_{1}, x_{2}, \ldots, x_{n}\right)$ with diagonal entries $x_{1}, \ldots, x_{n}$, and $X$ is viewed as a linear map from $N$ to $N$ via the $B$-action. Define the dual character of $N$ to be the character of the dual module $N^{*}$ :

$$
\begin{aligned}
\operatorname{char}^{*}(N)\left(x_{1}, \ldots, x_{n}\right) & =\operatorname{tr}\left(X: N^{*} \rightarrow N^{*}\right) \\
& =\operatorname{char}(N)\left(x_{1}^{-1}, \ldots, x_{n}^{-1}\right) .
\end{aligned}
$$

A special case of dual characters of flagged Weyl modules of diagrams are Schubert polynomials:

Theorem 5.2 [8] Let $w$ be a permutation, $D(w)$ be the Rothe diagram of $w$, and $\mathcal{M}_{D(w)}$ be the associated flagged Weyl module. Then,

$$
\mathfrak{S}_{w}=\operatorname{char}^{*} \mathcal{M}_{D(w)} .
$$

Another special family of dual characters of flagged Weyl modules of diagrams, for socalled skyline diagrams of compositions, are key polynomials [3].

Definition 5.3 For a diagram $D \subseteq[n] \times[n]$, let $\chi_{D}=\chi_{D}\left(x_{1}, \ldots, x_{n}\right)$ be the dual character

$$
\chi_{D}=\operatorname{char}^{*} \mathcal{M}_{D} \text {. }
$$

We now work towards proving Theorem 5.8. We start by reviewing some material from [4] for the reader's convenience. We then derive several lemmas that simplify the proof of Theorem 5.8.

Theorem 5.4 (cf. [4, Theorem 7]) For any diagram $D \subseteq[n] \times[n]$, the monomials appearing in $\chi_{D}$ are exactly

$$
\left\{\prod_{j=1}^{n} \prod_{i \in C_{j}} x_{i} \mid C \leq D\right\} .
$$

Proof (Following that of [4, Theorem 7]) Denote by $X$ the diagonal matrix $\operatorname{diag}\left(x_{1}, x_{2}, \ldots, x_{n}\right)$. First, note that $y_{i j}$ is an eigenvector of $X$ with eigenvalue $x_{i}^{-1}$. Take a diagram $C=$ $\left(C_{1}, \ldots, C_{n}\right)$ with $C \leq D$. Then, the element $\prod_{j=1}^{n} \operatorname{det}\left(Y_{D_{j}}^{C_{j}}\right)$ is an eigenvector of $X$ with eigenvalue $\prod_{j=1}^{n} \prod_{i \in C_{j}} x_{i}^{-1}$. Since $\mathcal{M}_{D}$ is spanned by elements $\prod_{j=1}^{n} \operatorname{det}\left(Y_{D_{j}}^{C_{j}}\right)$ and each is an eigenvector of $X$, the monomials appearing in the dual character $\chi_{D}$ are exactly $\left\{\prod_{j=1}^{n} \prod_{i \in C_{j}} x_{i} \mid C \leq D\right\}$. 
Corollary 5.5 Let $D \subseteq[n] \times[n]$ be a diagram. Fix any diagram $C^{(1)} \leq D$ and set

$$
\boldsymbol{m}=\prod_{j=1}^{n} \prod_{i \in C_{j}^{(1)}} x_{i}
$$

Let $C^{(1)}, \ldots, C^{(r)}$ be all the diagrams $C$ such that $C \leq D$ and $\prod_{j=1}^{n} \prod_{i \in C_{j}} x_{i}=\boldsymbol{m}$. Then, the coefficient of $\boldsymbol{m}$ in $\chi_{D}$ is equal to

$$
\operatorname{dim}\left(\operatorname{Span}_{\mathbb{C}}\left\{\prod_{j=1}^{n} \operatorname{det}\left(Y_{D_{j}}^{C_{j}^{(i)}}\right) \mid i \in[r]\right\}\right) .
$$

Proof The coefficient of $\boldsymbol{m}$ in $\chi_{D}$ equals the dimension of the eigenspace of $\boldsymbol{m}^{-1}$ in $\mathcal{M}_{D}$ $\left(\boldsymbol{m}^{-1}\right.$ occurs here instead of $\boldsymbol{m}$ since $\chi_{D}$ is the dual character of $\mathcal{M}_{D}$ ). This eigenspace equals

$$
\operatorname{Span}_{\mathbb{C}}\left\{\prod_{j=1}^{n} \operatorname{det}\left(Y_{D_{j}}^{C_{j}^{(i)}}\right) \mid i \in[r]\right\}
$$

so the result follows.

The understanding of the coefficients of the monomials of $\chi_{D}$ given in Corollary 5.5 is key to our proof of Theorem 5.8. We set up some notation now.

Given diagrams $C, D \subseteq[n] \times[n]$ and $k, l \in[n]$, let $\widehat{C}$ and $\widehat{D}$ denote the diagrams obtained from $C$ and $D$ by removing any boxes in row $k$ or column $l$. Fix a diagram $D$. For each diagram $\widehat{C}$, let

$$
\widehat{C}_{\text {aug }}=\widehat{C} \cup\{(k, i) \mid(k, i) \in D\} \cup\{(i, l) \mid(i, l) \in D\} \subseteq[n] \times[n] .
$$

The following lemma is immediate and its proof is left to the reader.

Lemma 5.6 Let $C, D \subseteq[n] \times[n]$ be diagrams and $k, l \in[n]$. If $\widehat{C} \leq \widehat{D}$, then $\widehat{C}_{\text {aug }} \leq D$. In particular, every diagram $C^{\prime} \leq \widehat{D}$ with no boxes in row $k$ can be obtained from some diagram $C \leq D$ by removing any boxes in row $k$ or column l from $C$.

The following result is our key lemma. For a polynomial $f \in \mathbb{Z}\left[x_{1}, \ldots, x_{n}\right]$ and a monomial $\boldsymbol{m}$, let $[\boldsymbol{m}] f$ denote the coefficient of $\boldsymbol{m}$ in $f$.

Lemma 5.7 Fix a diagram $D$ and $k, l \in[n]$. Let $\left\{\widehat{C}^{(i)}\right\}_{i \in[m]}$ be a set of diagrams with $\widehat{C}^{(i)} \leq \widehat{D}$ for each $i$, and denote $\widehat{C}_{\text {aug }}^{(i)}$ by $C^{(i)}$ for $i \in[m]$. If the polynomials $\left\{\prod_{j \in[n]} \operatorname{det}\left(Y_{D_{j}}^{C_{j}^{(i)}}\right)\right\}_{i \in[m]}$ are linearly dependent, then so are the polynomials $\left\{\prod_{j \in[n] \backslash\{l\}} \operatorname{det}\left(Y_{\widehat{D}_{j}^{j}}^{\widehat{C}_{j}^{(i)}}\right)\right\}_{i \in[m]}$.

Proof We are given that

$$
\sum_{i \in[m]} c_{i} \prod_{j \in[n]} \operatorname{det}\left(Y_{D_{j}}^{C_{j}^{(i)}}\right)=0
$$


for some constants $\left(c_{i}\right)_{i \in[m]} \in \mathbb{C}^{m}$ not all zero. Since $C^{(i)}=\widehat{C}_{\text {aug }}^{(i)}$ for $\widehat{C}^{(i)} \leq \widehat{D}$ we have that $C_{l}^{(i)}=D_{l}$ for every $i \in[m]$. Thus, (2) can be rewritten as

$$
\operatorname{det}\left(Y_{D_{l}}^{D_{l}}\right)\left(\sum_{i \in[m]} c_{i} \prod_{j \in[n] \backslash\{l\}} \operatorname{det}\left(Y_{D_{j}}^{C_{j}^{(i)}}\right)\right)=0 .
$$

However, since $\operatorname{det}\left(Y_{D_{l}}^{D_{l}}\right) \neq 0$, we conclude that

$$
\sum_{i \in[m]} c_{i} \prod_{j \in[n] \backslash\{l\}} \operatorname{det}\left(Y_{D_{j}}^{C_{j}^{(i)}}\right)=0 .
$$

First consider the case that the only boxes of $D$ in row $k$ or column $l$ are those in $D_{l}$. If this is the case then

$$
\prod_{j \in[n] \backslash\{l\}} \operatorname{det}\left(Y_{\widehat{D}_{j}}^{\widehat{C}_{j}^{(i)}}\right)=\prod_{j \in[n] \backslash\{l\}} \operatorname{det}\left(Y_{D_{j}}^{C_{j}^{(i)}}\right)
$$

for each $i \in[m]$. Therefore,

$$
\sum_{i \in[m]} c_{i} \prod_{j \in[n] \backslash\{l\}} \operatorname{det}\left(Y_{\widehat{D}_{j}}^{\widehat{C}_{j}^{(i)}}\right)=\sum_{i \in[m]} c_{i} \prod_{j \in[n] \backslash\{l\}} \operatorname{det}\left(Y_{D_{j}}^{C_{j}^{(i)}}\right) .
$$

Combining (4) and (6) we obtain that the polynomials $\left\{\prod_{j \in[n] \backslash\{l\}} \operatorname{det}\left(Y_{\widehat{D}_{j}}^{\widehat{C}_{j}^{(i)}}\right)\right\}_{i \in[m]}$ are linearly dependent, as desired.

Now, suppose that there are boxes of $D$ in row $k$ that are not in $D_{l}$. Let $j_{1}<\cdots<j_{p}$ be all indices $j \neq l$ such that $D_{j}=\widehat{D}_{j} \cup\{k\}$. Then also $C_{j_{q}}^{(i)}=\widehat{C}_{j_{q}}^{(i)} \cup\{k\}$ for each $i \in[m]$ and $q \in[p]$. Let us consider the left-hand side of (4) as a polynomial in $y_{k k}$. Then, (4) implies that the coefficient of $y_{k k}^{p}$ is 0 :

$$
\left[y_{k k}^{p}\right] \sum_{i \in[m]} c_{i} \prod_{j \in[n] \backslash\{l\}} \operatorname{det}\left(Y_{D_{j}}^{C_{j}^{(i)}}\right)=0 .
$$

However,

$$
\left[y_{k k}^{p}\right] \prod_{j \in[n] \backslash\{l\}} \operatorname{det}\left(Y_{D_{j}}^{C_{j}^{(i)}}\right)=\prod_{j \in[n] \backslash\{l\}} \operatorname{det}\left(Y_{\widehat{D}_{j}}^{\widehat{C}_{j}^{(i)}}\right),
$$

as is seen by Laplace expansion on the $k$ th row, and therefore

$$
\left[y_{k k}^{p}\right] \sum_{i \in[m]} c_{i} \prod_{j \in[n] \backslash\{l\}} \operatorname{det}\left(Y_{D_{j}}^{C_{j}^{(i)}}\right)=\sum_{i \in[m]} c_{i} \prod_{j \in[n] \backslash\{l\}} \operatorname{det}\left(Y_{\widehat{D}_{j}}^{\widehat{C}_{j}^{(i)}}\right) .
$$

Thus, (7) and (9) imply that

$$
\sum_{i \in[m]} c_{i} \prod_{j \in[n] \backslash\{l\}} \operatorname{det}\left(Y_{\widehat{D}_{j}}^{\widehat{C}_{j}^{(i)}}\right)=0,
$$

as desired.

We now state and prove Theorem 1.2 and its generalization Theorem 5.8. 
Theorem 5.8 Fix a diagram $D \subseteq[n] \times[n]$ and let $\widehat{D}$ be the diagram obtained from $D$ by removing any boxes in row $k$ or column $l$. Then

$$
\chi_{D}\left(x_{1}, \ldots, x_{n}\right)=M\left(x_{1}, \ldots, x_{n}\right) \chi_{\widehat{D}}\left(x_{1}, \ldots, x_{k-1}, 0, x_{k+1}, \ldots, x_{n}\right)+F\left(x_{1}, \ldots, x_{n}\right),
$$

where $F\left(x_{1}, \ldots, x_{n}\right) \in \mathbb{Z}_{\geq 0}\left[x_{1}, \ldots, x_{n}\right]$ and

$$
M\left(x_{1}, \ldots, x_{n}\right)=\left(\prod_{(k, i) \in D} x_{k}\right)\left(\prod_{(i, l) \in D} x_{i}\right) .
$$

Proof Let $M=M\left(x_{1}, \ldots, x_{n}\right)$. We must show that $[M \boldsymbol{m}] \chi_{D} \geq[\boldsymbol{m}] \chi_{\widehat{D}}$ for each monomial $\boldsymbol{m}$ of $\chi_{\widehat{D}}$ not divisible by $x_{k}$. Let $C^{(1)}, \ldots, C^{(r)}$ be all the diagrams $C$ such that $C \leq D$ and $\prod_{j=1}^{n} \prod_{i \in C_{j}} x_{i}=M \boldsymbol{m}$. By Corollary 5.5,

$$
[M \boldsymbol{m}] \chi_{D}=\operatorname{dim}\left(\operatorname{Span}_{\mathbb{C}}\left\{\prod_{j=1}^{n} \operatorname{det}\left(Y_{D_{j}}^{C_{j}^{(i)}}\right) \mid i \in[r]\right\}\right) .
$$

Let $1,2, \ldots, q$ be the indices of the distinct diagrams among $\widehat{C}^{(1)}, \ldots, \widehat{C}^{(r)}$. By Lemma 5.6, $\widehat{C}^{(1)}, \ldots, \widehat{C}^{(q)}$ are all the diagrams $C$ such that $C \leq \widehat{D}$ and $\prod_{j=1}^{n} \prod_{i \in C_{j}} x_{i}=\boldsymbol{m}$, as no diagram with this dual eigenvalue can have a box in row $k$. So Corollary 5.5 implies that

$$
[\boldsymbol{m}] \chi_{\widehat{D}}=\operatorname{dim}\left(\operatorname{Span}_{\mathbb{C}}\left\{\prod_{j=1}^{n} \operatorname{det}\left(Y_{\widehat{D}_{j}}^{\widehat{C}_{j}^{(i)}}\right) \mid i \in[q]\right\}\right) .
$$

Finally, Lemma 5.7 implies that

$\operatorname{dim}\left(\operatorname{Span}_{\mathbb{C}}\left\{\prod_{j=1}^{n} \operatorname{det}\left(Y_{D_{j}}^{C_{j}^{(i)}}\right) \mid i \in[r]\right\}\right) \geq \operatorname{dim}\left(\operatorname{Span}_{\mathbb{C}}\left\{\prod_{j=1}^{n} \operatorname{det}\left(Y_{\widehat{D}_{j}}^{\widehat{C}_{j}^{(i)}}\right) \mid i \in[q]\right\}\right)$, so $[M \boldsymbol{m}] \chi_{D} \geq[\boldsymbol{m}] \chi_{\widehat{D}}$ for each monomial $\boldsymbol{m}$ of $\chi_{\widehat{D}}$ not divisible by $x_{k}$; that is

$$
\chi_{D}\left(x_{1}, \ldots, x_{n}\right)-M \chi_{\widehat{D}}\left(x_{1}, \ldots, x_{k-1}, 0, x_{k+1}, \ldots, x_{n}\right) \in \mathbb{Z}_{\geq 0}\left[x_{1}, \ldots, x_{n}\right] .
$$

1.2 Fix $w \in S_{n}$ and let $\sigma \in S_{n-1}$ be the pattern with Rothe diagram $D(\sigma)$ obtained by removing row $k$ and column $w_{k}$ from $D(w)$. Then

$$
\mathfrak{S}_{w}\left(x_{1}, \ldots, x_{n}\right)=M\left(x_{1}, \ldots, x_{n}\right) \mathfrak{S}_{\sigma}\left(x_{1}, \ldots, \widehat{x}_{k}, \ldots, x_{n}\right)+F\left(x_{1}, \ldots, x_{n}\right),
$$

where $F \in \mathbb{Z}_{\geq 0}\left[x_{1}, \ldots, x_{n}\right]$ and

$$
M\left(x_{1}, \ldots, x_{n}\right)=\left(\prod_{(k, i) \in D(w)} x_{k}\right)\left(\prod_{\left(i, w_{k}\right) \in D(w)} x_{i}\right) .
$$

Proof Specialize Theorem 5.8 to the case that $D$ is a Rothe diagram $D(w)$ and $l=w_{k}$. The dropping of $x_{k}$ is due to reindexing, since the entirety of row $k$ and column $w_{k}$ of $D(w)$ are removed from to obtain $D(\sigma)$, not just the boxes in row $k$ and column $w_{k}$.

Corollary 5.9 Fix $w \in S_{n}$ and let $\sigma \in S_{m}$ be any pattern contained in $w$. If $k$ is a coefficient of a monomial in $\mathfrak{S}_{\sigma}$, then $\mathfrak{S}_{w}$ contains a monomial with coefficient at least $k$.

Proof Immediate consequence of repeated applications of Theorem 1.2. 
Acknowledgements We are grateful to Sara Billey and Allen Knutson for many discussions about Schubert polynomials. We thank the Institute for Advanced Study for providing a hospitable environment for our collaboration.

Open Access This article is licensed under a Creative Commons Attribution 4.0 International License, which permits use, sharing, adaptation, distribution and reproduction in any medium or format, as long as you give appropriate credit to the original author(s) and the source, provide a link to the Creative Commons licence, and indicate if changes were made. The images or other third party material in this article are included in the article's Creative Commons licence, unless indicated otherwise in a credit line to the material. If material is not included in the article's Creative Commons licence and your intended use is not permitted by statutory regulation or exceeds the permitted use, you will need to obtain permission directly from the copyright holder. To view a copy of this licence, visit http://creativecommons.org/licenses/by/4.0/.

\section{References}

1. Bergeron, N., Billey, S.: RC-graphs and Schubert polynomials. Exp. Math. 2(4), 257-269 (1993)

2. Billey, S., Jockusch, W., Stanley, R.P.: Some combinatorial properties of Schubert polynomials. J. Algebraic Combin. 2(4), 345-374 (1993)

3. Demazure, M.: Une nouvelle formule des caracteres. J. Combin. Theory Ser. A 70(1), 107-143 (1995)

4. Fink, A., Mészáros, K., St, A., Dizier, : Schubert polynomials as integer point transforms of generalized permutahedra. Adv. Math. 332, 465-475 (2018)

5. Fomin, S., Kirillov, A.N.: The Yang-Baxter equation, symmetric functions, and Schubert polynomials. Discrete Math. 153(1), 123-143 (1996). (Proceedings of the 5th Conference on Formal Power Series and Algebraic Combinatorics)

6. Fomin, S., Stanley, R.P.: Schubert polynomials and the nilCoxeter algebra. Adv. Math. 103(2), 196-207 (1994)

7. Knutson, A., Miller, E.: Gröbner geometry of Schubert polynomials. Ann. Math. (2) 161(3), 1245-1318 (2005)

8. Kraśkiewicz, W., Pragacz, P.: Foncteurs de Schubert. C. R. Acad. Sci. Paris Sér. I Math. 304(9), 209-211 (1987)

9. Lam, T., Lee, S., Shimozono, M.: Back stable Schubert calculus, Jun 2018. arxiv: 1806.11233

10. Lascoux, A., Schützenberger, M.-P.: Polynômes de Schubert. C. R. Acad. Sci. Paris Sér I Math 294(13), 447-450 (1982)

11. Lascoux, A., Schützenberger, M.-P.: Keys and standard bases. IMA Math. Appl. 19, 125-144 (1990)

12. Lenart, C.: A unified approach to combinatorial formulas for Schubert polynomials. J. Algebraic Combin. 20(3), 263-299 (2004)

13. Magyar, P.: Schubert polynomials and Bott-Samelson varieties. Comment. Math. Helv. 73(4), 603-636 (1998)

14. Manivel, L.: Symmetric functions, Schubert polynomials and degeneracy loci, volume 6 of SMF/AMS Texts and Monographs. American Mathematical Society, Providence, RI; Société Mathématique de France, Paris, 2001. Translated from the 1998 French original by John R. Swallow, Cours Spécialisés [Specialized Courses], 3

15. Monical, C., Tokcan, N., Yong, A.: Newton polytopes in algebraic ombinatorics. Selecta Math. (N.S.) 25, 66 (2019)

16. Reiner, V., Shimozono, M.: Key polynomials and a flagged Littlewood-Richardson rule. J. Combin. Theory Ser. A 70(1), 107-143 (1995)

17. Weigandt, A., Yong, A.: The prism tableau model for Schubert polynomials. J. Comb. Theory Ser. A 154, 551-582 (2018)

Publisher's Note Springer Nature remains neutral with regard to jurisdictional claims in published maps and institutional affiliations. 